Research Article

\title{
Chronic Ethanol Consumption Induces Osteopenia via Activation of Osteoblast Necroptosis
}

\author{
Man Guo $\mathbb{D}^{1,2,3}$ Yong-Li Huang ${ }^{1},{ }^{4}$ Qi Wu $\mathbb{D},{ }^{5}$ Li Chai ${ }^{5},{ }^{5}$ Zong-Zhe Jiang, ${ }^{2,3,6}$ \\ Yan Zeng $\mathbb{D}^{1,2,3}$ Sheng-Rong Wan $\mathbb{D}^{1,2,3}$ Xiao-Zhen Tan, ${ }^{2,3,6}$ Yang Long $\mathbb{D},{ }^{2,3,6}$ \\ Jun-Ling Gu $\mathbb{D}^{7}$ Fang-Yuan Teng $\mathbb{D}^{2,3,6}$ and Yong $X u \mathbb{D}^{1,2,3}$ \\ ${ }^{1}$ Department of Endocrinology and Metabolism, The Affiliated Hospital of Southwest Medical University, Luzhou, \\ Sichuan 646000, China \\ ${ }^{2}$ Sichuan Clinical Research Center for Nephropathy, Luzhou, Sichuan 646000, China \\ ${ }^{3}$ Cardiovascular and Metabolic Diseases Key Laboratory of Luzhou, Luzhou, Sichuan 646000, China \\ ${ }^{4}$ Department of Outpatient, The Affiliated Hospital of Southwest Medical University, Luzhou, Sichuan 646000, China \\ ${ }^{5}$ Department of Pathology, The Affiliated Hospital of Southwest Medical University, Luzhou, Sichuan 646000, China \\ ${ }^{6}$ Experimental Medicine Center, The Affiliated Hospital of Southwest Medical University, Luzhou, Sichuan 646000, China \\ ${ }^{7}$ Department of Endocrinology, Yibin Second People's Hospital, Yibin, Sichuan 644000, China
}

Correspondence should be addressed to Fang-Yuan Teng; teng199009@163.com and Yong Xu; xywyll@aliyun.com

Received 17 May 2021; Revised 14 September 2021; Accepted 4 October 2021; Published 27 October 2021

Academic Editor: Antonio Desmond McCarthy

Copyright (c) 2021 Man Guo et al. This is an open access article distributed under the Creative Commons Attribution License, which permits unrestricted use, distribution, and reproduction in any medium, provided the original work is properly cited.

\begin{abstract}
Chronic high-dose alcohol consumption impairs bone remodeling, reduces bone mass, and increases the risk of osteoporosis and bone fracture. However, the mechanisms underlying alcohol-induced osteoporosis are yet to be elucidated. In this study, we showed that excess intake of ethyl alcohol (EtOH) resulted in osteopenia and osteoblast necroptosis in mice that led to necrotic lesions and reduced osteogenic differentiation in bone marrow mesenchymal stem cells (BMMSCs). We found that EtOH treatment led to the activation of the RIPK1/RIPK3/MLKL signaling, resulting in increased osteoblast necroptosis and decreased osteogenic differentiation and bone formation both in vivo and in vitro. We further discovered that excessive EtOH treatment-induced osteoblast necroptosis might partly depend on reactive oxygen species (ROS) generation; concomitantly, ROS contributed to necroptosis of osteoblasts through a positive feedback loop involving RIPK1/RIPK3. In addition, blocking of the RIPK1/RIPK3/MLKL signaling by necrostatin-1 (Nec-1), a key inhibitor of RIPK1 kinase in the necroptosis pathway, or antioxidant $\mathrm{N}$-acetylcysteine (NAC), an inhibitor of ROS, could decrease the activation of osteoblast necroptosis and ameliorate alcohol-induced osteopenia both in vivo and in vitro. Collectively, we demonstrated that chronic high-dose alcohol consumption induced osteopenia via osteoblast necroptosis and revealed that RIPK1 kinase may be a therapeutic target for alcohol-induced osteopenia.
\end{abstract}

\section{Introduction}

Chronic and excessive alcohol consumption has harmful effects on multiple organs, including the brain, heart, liver, and skeleton [1-4]. Chronic heavy alcohol consumption is recognized as a major cause of secondary osteoporosis and is positively associated with impaired bone remodeling, extensive bone loss, and fracture [5-7]. Multiple mechanisms are responsible for alcohol-induced osteoporosis (AOP), including a direct effect on bone formation and bone resorption $[8,9]$. Ethyl alcohol (EtOH) intake inhibits bone marrow mesenchymal stem cell- (BMMSC-) derived osteoblast proliferation and enhances osteoclast differentiation via the RANKL/RANKL/OPG signaling pathway that promotes bone resorption in long-term alcohol users $[10,11]$. The imbalanced osteo/adipogenic lineage commitment of BMMSCs is modulated via activation of the PI3K/AKT $/ \mathrm{m}$ TOR signaling that results in downregulation of runt- 
related transcription factor 2 (Runx2) and upregulation of peroxisome proliferator-activated receptor $\gamma$ (PPAR $\gamma)$ [12-14]. The indirect action of chronic alcohol-induced bone loss may also be regulated by inducing oxidative stress [13, 15]. It has been reported that with alcohol exposure, increased levels of reactive oxygen species (ROS) may directly precede the production of TNF- $\alpha$ and other inflammatory cytokines in the bone marrow and contribute to bone formation decrease [16]. Furthermore, increased endothelial nitric oxide synthase (eNOS) and overexpressed inducible nitric oxide synthase (iNOS) in the bone may contribute to osteocyte apoptosis and necrosis [17].

Apoptosis was identified as the early stage in alcoholmediated osteoporosis, specifically an increase in osteoblast apoptosis and osteocytes $[18,19]$, while necrosis occurs in succession following extensive apoptosis. Deficiency or inhibition of the aspartate-specific cysteine protease- 8 (caspase8) can switch cell death into a specific form of necrosis, resulting in necroptosis [20-22]. Necroptosis, having typical morphological characteristics of necrosis, can be induced by tumor necrosis factor- $\alpha$ (TNF- $\alpha$ ), a factor associated with suicide (Fas, which binds to the ligand CD95L, known as FASL), TNF-related apoptosis-inducing ligand (TRAIL), and other death cytokines [23-25]. Receptor-interacting serine/threonine protein kinase 1 (RIPK1) and 3 (RIPK3) activate the mixed lineage kinase domain-like protein (MLKL), which then recruits the necrosome to localize on the cell membrane and causes cell necroptosis [26, 27]. Furthermore, necroptosis can be regulated and pharmacologically inhibited by necrostatin-1 (Nec-1), the first small-molecule inhibitor of RIPK1 kinase to be developed [28-30]. Nec-1 is a cellular protector and has been widely used to investigate the role of RIPK1 in multiple disease models, including neurodegenerative and cerebrovascular diseases [31-33], atherosclerosis [34], heart attack [35, 36], and liver, retinal, and renal injury $[37,38]$. Nec-1 has been reported to inhibit the death of chondrocytes in cartilage injury and promote cartilage repair [39]. Treatment with $\mathrm{Nec}-1$ also reduces osteocyte necrosis and increases bone formation in glucocorticoidinduced osteoporosis (GIOP) rats and ovariectomized rats that resulted in the kinase RIPK1-dependent necroptosis [40, 41]. In addition, the necroptosis signaling pathway is also found to be involved in the pathophysiology of osteosarcoma [42]. However, it remains unknown whether RIPK1 mediates damage via activation of necroptosis in an alcohol-induced osteopenia model and the effect of necrostatin-1 on osteopenia requires further elucidation.

In the current study, we investigated the effect of ethanol treatment on osteogenesis and osteopenia and the underlying mechanisms that mediate necroptosis in alcoholinduced osteopenia. Our investigations revealed that EtOH treatment increased osteoblast necroptosis and decreased osteogenic differentiation and bone formation in vivo; ROS have been considered, at least in part, as a critical driving force for necroptosis; necrostatin-1 and $\mathrm{N}$-acetylcysteine (NAC) ameliorated osteopenia via inhibition of the RIPK1/RIPK3/MLKL signaling pathways. Therefore, RIPK1 kinase and targeted antioxidants may be therapeutic targets for alcohol-induced osteopenia.

\section{Materials and Methods}

2.1. Animals. All animal experimental protocols were approved by the Animal Research Center of Southwest Medical University (No.: 20210928-007) and performed in accordance with the policies of Chinese animal research committees. Male C57BL/6J mice at 8 weeks were purchased from the SPF (Beijing) Biotechnology Co., Ltd. (China, SCXK 2016-0002) and acclimatized for two weeks with temperature and humidity controlled at $21 \pm 0.5^{\circ} \mathrm{C}$ and 55 $\pm 10 \%$, respectively. Then, mice were randomly divided into a control vehicle-treated (control), necrostatin-1 drug control group (Nec-1), chronic high-dose alcohol group $(\mathrm{EtOH})$, and chronic high-dose alcohol add on necrostatin1 treatment group ( $\mathrm{EtOH}+\mathrm{Nec}-1)(n=15 /$ group$)$. Mice were administered $0.2 \mathrm{ml} / 10 \mathrm{~g} / \mathrm{d}$ of alcohol (gradually increase concentration to $30 \% v / v)$ via gavage $[43,44]$ and $1.65 \mathrm{mg} / \mathrm{kg} / \mathrm{d}$ of necrostatin-1 (Sigma-Aldrich, St. Louis, USA) by intraperitoneal injection (ip) [40]; the control received the $\mathrm{ddH}_{2} \mathrm{O}$ and $1000 \mu \mathrm{l} / \mathrm{kg} / \mathrm{d}$ of $1 \%$ DMSO solution (Sigma-Aldrich, St. Louis, USA) in the same volume by the same route for 22 weeks.

2.2. Dual-Energy X-Ray Absorptiometry (DXA). Mice were anesthetized with $1 \%$ pentobarbital sodium $(50 \mathrm{mg} / \mathrm{kg}$ body weight, ip). The whole body, lumbar spine, and bilateral femur scans were analyzed using the region of interest (ROI) tools (GE Healthcare Lunar, Germany). Bone mineral density (BMD) was automatically calculated for each ROI scan. A quality assurance scan of the calibration block provided with the scanner was performed daily.

2.3. Micro-Computed Tomography Assay (Micro-CT). The distal femurs were harvested, fixed in $4 \%$ paraformaldehyde (PFA; Sigma-Aldrich, St. Louis, USA), and placed in fresh $70 \%$ ethanol. The specimens were then scanned using a high-resolution $\mu \mathrm{CT}$ scanner (SCANCO Medical AG, $\mu \mathrm{CT}$ 50, Brüttiselen, Switzerland) with a voxel size of $15 \mu \mathrm{m}$, an $\mathrm{X}$-ray tube voltage of $70 \mathrm{kVp}$, a current of $200 \mu \mathrm{A}$, and an integration time of $300 \mathrm{~ms}$. The scanner special software (SCANCO Medical Evaluation) was used to locate and analyze the distal femurs. The volumes-of-interest (VOIs) of bone tissue were $29 \times 29 \times 29 \mu \mathrm{m}^{3}$, and a total of 1800 2000 layers of cancellous bone tissue were defined as the final ROI. The datasets were loaded onto the Amira 5.3.1 software (Visage Imaging, Berlin, Germany) for visualization and analysis. In addition, BMD, bone volume/total volume (BV/TV), trabecular thickness (Tb.Th), trabecular number (Tb.N), trabecular separation (Tb.Sp), cortical thickness (Ct.Th), and 3D reconstructions for each specimen were calculated using the Amira software with 245 analysis thresholds.

2.4. Histological and Immunofluorescent and Immuno histochemistry Staining. The femurs were fixed in 4\% PFA for $24 \mathrm{~h}$, decalcified with $10 \%$ ethylenediaminetetraacetic acid (EDTA, pH 7.6-7.8) for 40 days at room temperature, and embedded in paraffin. Sections of $2 \mu \mathrm{m}$ thickness were deparaffinized, dehydrated, and stained with hematoxylin and eosin (H\&E) and TRACP staining for histological and 
histomorphometric analysis (Leica, Germany). Bone remodeling activity was evaluated by the ratio of osteoid surface to bone surface (OS/BS, \%), osteoblast surface to bone surface (Ob.S/BS, \%), and eroded surface to bone surface (ES/BS, $\%$ ), according to the recommendations of the ASBMR Histomorphometry Nomenclature Committee [45]. Sections of $4 \mu \mathrm{m}$ thickness were subjected to immunofluorescent and immunohistochemistry staining, blocked with $10 \%$ goat serum (Gibco, USA), and incubated with Runx2- or RIPK1or RIPK3- or cleaved caspase-3-specific antibodies $(1: 100$; Abcam, Cambridge, UK) at $4^{\circ} \mathrm{C}$ overnight, followed by a fluorescent secondary antibody $(1: 200$; Cell Signaling Technology, USA) for immunofluorescent staining; treated with secondary antibody, incubated with the streptavidinhorseradish peroxidase complex, visualized with diaminobenzidine, and counterstained with hematoxylin for immunohistochemistry staining. The trabecular bone structure parameters and area percentage and the percentage of Runx2-, RIPK1-, and RIPK3-positive cells were calculated using the Image-Pro Plus software.

2.5. Isolation and Culturing of Mouse BMMSCs. Mouse BMMSCs were isolated from the bilateral femoral marrow of six-week-old male C57BL/6J mice. Single-cell suspensions were cultured in alpha-minimum essential medium $(\alpha$ MEM; Hyclone, Logan, UT, USA) containing 15\% fetal bovine serum (FBS; Gibco, Grand Island, NY, USA), 1\% penicillin/streptomycin (Invitrogen, Grand Island, NY, USA), $2 \mathrm{mM}$ L-glutamine (Sigma-Aldrich, St. Louis, USA), and $55 \mu \mathrm{M}$ 2-mercaptoethanol (Sigma-Aldrich, St. Louis, USA) in a $5 \% \mathrm{CO}_{2}$ humidified atmosphere at $37^{\circ} \mathrm{C}$. Nonadherent cells were discarded after $48 \mathrm{~h}$, and the adherent BMMSCs were cultured for 16 days until colonies are formed and then passaged once for further experimental use. The essential medium was changed every 2 days.

2.6. Flow Cytometry. Flow cytometry was used to test the surface markers of the isolated BMMSCs at passage three. Adherent cells were collected and incubated with a positive surface epitope profile with monoclonal antibodies for mouse CD105 and Scal and negative cell surface markers with CD34 and CD45 (FITC anti-mouse CD34, CD45, CD105, and Sca1 antibodies; BD Biosciences, USA) and then were identified by flow cytometry analysis (BD Biosciences, San Jose, CA, USA), and the data were analyzed with EPICS $\mathrm{XL}$ software.

\subsection{Osteogenic Differentiation and Mineralization Assays.} The flow cytometry was used to identify BMMSCs in all experiments between three and seven passages. After the BMMSCs cultured in essential medium reached $70 \%$ confluency, the medium was replaced with an osteogenic medium containing essential medium and supplemented with $2 \mathrm{mM} \beta$-glycerophosphate (Sigma-Aldrich, St. Louis, USA), $100 \mu \mathrm{M}$ L-ascorbic acid 2-phosphate (Sigma-Aldrich, St. Louis, USA), and $10 \mathrm{nM}$ dexamethasone (Sigma-Aldrich, St. Louis, USA).

Identified BMMSCs $\left(1 \times 10^{5}\right)$ and mouse preosteoblastic MC3T3-E1 cells purchased from American Type Culture
Collection (ATCC, USA) were seeded in 6-well plates with the osteogenic medium for 7 days. Alkaline phosphatase (ALP) staining (Beyotime Institute of Biotechnology, Shanghai, China) was performed using a standard protocol after 7 days of induction in 24-well plates. Further, alizarin red staining (Beyotime Institute of Biotechnology, Shanghai, China) was performed after culturing in osteogenic medium for 28 days to detect mineralized nodule formation. Images were captured with a converted microscope (Olympus, Tokyo, Japan), and quantitative analysis was performed with the Image-Pro Plus software.

2.8. Cell Proliferation Assay. The effects of ethanol or necrostatin-1 on cell proliferation were evaluated using the Cell Counting Kit- 8 assay (Beyotime Institute of Biotechnology, Shanghai, China). Briefly, BMMSCs and MC3T3-E1 $\left(1 \times 10^{5}\right.$ cells/well $)$ were seeded in 96-well plates and cultured in $100 \mu \mathrm{l}$ growth medium until the cells proliferated to about $30 \%$, respectively. The cells were then starved for $6 \mathrm{~h}$, and the medium was replaced with osteogenic differentiation induction medium, and different concentrations of alcohol were added at 24,48 , and $72 \mathrm{~h}$, respectively. After alcohol stimulation, $10 \mu \mathrm{l}$ CCK-8 solution and $90 \mu \mathrm{l}$ fresh medium were added to each well, with 5 repeat wells, and incubated at $37^{\circ} \mathrm{C}$ for $2 \mathrm{~h}$, according to the manufacturer's protocol. Cell proliferation was assessed by measuring the absorbance at a wavelength of $450 \mathrm{~nm}$ (Thermo Fisher Scientific, MA, USA), and the appropriate time and concentration of alcohol intervention were determined. The same method was followed for necrostatin-1 $(50 \mu \mathrm{mol} / \mathrm{l})$.

2.9. Real-Time PCR Analysis. Cells were cultured in 6-well plates with osteogenic medium and treated with alcohol $(150 \mathrm{mM})$ and necrostatin-1 $(50 \mu \mathrm{M})$ for $48 \mathrm{~h}$ to detect the gene expression of necroptosis markers (RIPK1, RIPK3, and MLKL), treated for 7 days to test the expression of Runx2, then extracted total RNA using TRIzol reagent (Qiagen, Valencia, CA, USA), and assessed the concentration and purity by a spectrophotometer. Reverse transcriptase reactions were completed by ReverTra Ace ${ }^{\circledR}$ qPCR RT Kit (Toyobo, Japan), and real-time PCR was performed using SYBR ${ }^{\circledR}$ Premix Ex Taq (2x) (Toyobo, Japan). Finally, the CT values were calculated in relation to $\beta$-actin CT values $(\mathrm{RQ}=-\Delta \Delta \mathrm{Ct})$. Primers were synthesized by Primer Premier 5.0 software, using Primer-BLAST to confirm the definition of primers in NCBI website, and synthesized by Shanghai Biotechnology Co., Ltd. (Supplementary Table S1). The same method also applied to examine the gene expression of mouse bone tissue.

2.10. Western Blot Analysis. BMMSCs were cultured and treated as described previously, and proteins were harvested with RIPA cell lysis buffer system (Cell Signaling Technology, USA) supplied with phosphatase inhibitors. Total protein concentrations were quantified by BCA protein assay kit (Beyotime Institute of Biotechnology, Shanghai, China). Equal amounts of proteins $(20 \mu \mathrm{g})$ were separated on $10 \%$ SDS-PAGE for protein electrophoresis and transferred to $0.2 \mu \mathrm{m}$ polyvinylidene fluoride (PVDF) membranes, which 
were blocked with 5\% nonfat dry milk for $1 \mathrm{~h}$ at room temperature and incubated at $4^{\circ} \mathrm{C}$ overnight with primary antibodies to mouse Runx2, RIPK1, RIPK3, MLKL, phosphor-RIPK3 (p-RIPK3), phosphor-MLKL (p-MLKL), cleaved caspase- 3 , and $\beta$-actin ( $1: 1000$; Abcam, Cambridge, UK) and phosphor-RIPK1 (p-RIPK1; $1: 1000$; Cell Signaling Technology, USA). After washing three times in PBST and incubated for 1 hour with HRP-conjugated second antibody at room temperature $(1: 2000$, Abcam, Cambridge, UK), immunoreactive protein was detected using enhanced chemiluminescence reagents (Millipore, USA) and immunoblots were quantified with ImageJ software. MC3T3-E1 were cultured with osteogenic differentiation induction medium and treated as described previously to verify EtOH-induced osteoblast necroptosis.

2.11. Double-Label Immunofluorescent Staining. After $48 \mathrm{~h}$ of intervention on confocal dishes, cells were fixed with $4 \%$ PFA for $30 \mathrm{~min}$, permeabilized in $0.5 \%$ Triton X-100 for $10 \mathrm{~min}$, and incubated with primary antibodies of antimouse RIPK1 (1:100; Abcam, Cambridge, UK) and antirabbit RIPK3 (1:100; Abcam, Cambridge, UK) at $4^{\circ} \mathrm{C}$ for overnight and then incubated with fluorescent goat antimouse and goat anti-rabbit secondary antibodies $(1: 200$; Abcam, Cambridge, UK) for 4 hours at room temperature. After stained with DAPI, acquired confocal images by OLYMPUS inverted laser scanning confocal microscope (Olympus, Tokyo, Japan) and mounted the positive coexpression of RIPK1 and RIPK3 rates.

2.12. Transmission Electron Microscopy (TEM). After EtOH treatment for $48 \mathrm{~h}$, the specimens were fixed in $2.5 \%$ glutaraldehyde for $2 \mathrm{~h}$ and postfixed in $1 \%$ osmium tetroxide for $90 \mathrm{~min}$ at $4^{\circ} \mathrm{C}$, dehydrated in ethanol, and embedded in epoxy resin. The cells were examined under a transmission electron microscope (H-7700, Hitachi, Japan).

2.13. Intracellular Reactive Oxygen Species Assay. After $\mathrm{EtOH}$ treatment for $48 \mathrm{~h}$, the ROS generation of MC3T3E1 was detected by a ROS detection kit (Beyotime Institute of Biotechnology, Shanghai, China). The fluorescence of $2^{\prime}$ $-7^{\prime}$-dihydrodichlorofluoroscein diacetate (DCFH-DA) was determined using a spectrofluorophotometer, and the mean fluorescence intensity (MFI) was analyzed by flow cytometry.

2.14. ELISA Assay. Peripheral blood serum was collected; serum alcohol concentration was measured with mouse EnzyChrom $^{\mathrm{TM}}$ Ethanol Assay Kit (BioAssay Systems, USA). TNF- $\alpha$ level was analyzed using mouse tumor necrosis factor- $\alpha$ ELISA kit (Andy Gene, China) with mouse peripheral blood serum and cell culture medium, according to the manufacturer's instructions. BALP, C-terminal telopeptide of type I collagen (CTX-I), tartrate-resistant acid phosphatase $5 \mathrm{~b}$ (TRACP $5 \mathrm{~b}$ ) levels, and proinflammatory cytokines (IL$1 \beta$ and IL-6) were analyzed using mouse ELISA kit (Andy Gene, China) with mouse peripheral blood serum.

2.15. Statistical Analysis. All experimental data were expressed as the mean \pm standard deviation (SD) from at least three independent experiments. Comparisons between the two groups were analyzed using independent two-tailed Student's $t$-test, and comparisons between multiple groups were accomplished using one-way ANOVA with the Bonferroni adjustment with SPSS 22.0 software (SPNN Inc., Chicago, IL, USA). $p$ values $<0.05$ were considered statistically significant.

\section{Results}

3.1. EtOH Treatment Leads to Osteogenic Differentiation Reduction and Osteopenia. In order to verify the effects of alcohol treatment on bone tissue, C57BL/6J male mice were administered via gavage with $0.2 \mathrm{ml} / 10 \mathrm{~g} / \mathrm{d}(30 \% \mathrm{v} / \mathrm{v})$ of alcohol for 22 weeks. All the animals were alive with no complications, and there was a significant difference in the body weight between the control group and $\mathrm{EtOH}$-treated group after administration of alcohol for 18 weeks $(p<0.05$, Figure 1(a)). The serum alcohol concentration of the mice in the EtOH-treated group was twice as much as the control group when sacrificed $(p<0.05)$, indicating the success of alcohol gavage (Figure 1(b)). BALP, an early marker of osteoblastic differentiation, in the serum was significantly decreased in the $\mathrm{EtOH}$-treated group compared to that in the control group $(p<0.05$, Figure $1(\mathrm{c}))$. Moreover, chronic alcohol consumption significantly reduced the bone mineral density (BMD) and mineral content of bilateral femurs, as measured by DXA $(p<0.05$, Figures $1(\mathrm{~d})$ and $1(\mathrm{e})$ ), and markedly decreased the BMD, BV/TV, Tb.Th, Tb.N and Ct.Th, concomitantly, increased the Tb.Sp by micro-CT analysis $(p<0.05$, Figures $1(\mathrm{f})$ and $1(\mathrm{~g}))$. Histomorphometric analysis performed on the H\&E stained sections revealed that the distal trabecular bone structure was disordered and loose, and the numbers of trabecular bone (yellow rectangle area, $p<0.01$ ) and the ratio of OS/BS (two yellow dotted lines) and Ob.S/BS were significantly decreased $(p<0.05)$; the ratio of ES/BS (two green dotted lines) was increased $(p<0.01)$ compared to untreated mice (Figures $1(\mathrm{~h})$ and $1(\mathrm{i})$ ). Immunofluorescent staining, western blotting, and RT-PCR detected the expression of Runx2, a specific marker of osteogenic differentiation, and the results revealed that mRNA and protein levels of Runx2 were significantly decreased (Figures $1(j)-1(n)$ ). Thus, this indicated that chronic alcohol consumption can lead to osteogenesis reduction and osteopenia in mice.

3.2. EtOH Treatment Leads to Osteoblast Necroptosis. To investigate the cause of EtOH-induced osteogenesis reduction and osteopenia, we detected necroptosis signaling. Interestingly, we found that high doses of $\mathrm{EtOH}$ markedly elevated the osteoblast necroptosis of bone tissue, as located and evidenced by the increased number of RIPK1-positive cells (Figures 2(a) and 2(b)) and RIPK3-positive cells (Figures 2(c) and 2(d)). The mRNA levels of RIPK1 and RIPK3 were correspondingly increased (Figures 2(e) and 2(f)). The levels of bone resorption markers, CTX-I and TRACP $5 b$, in serum $(p<0.05$, Supplementary Figure S1a, b) and the number of osteoclasts in the femurs identified by TRACP staining increased in the EtOH-treated mice. 


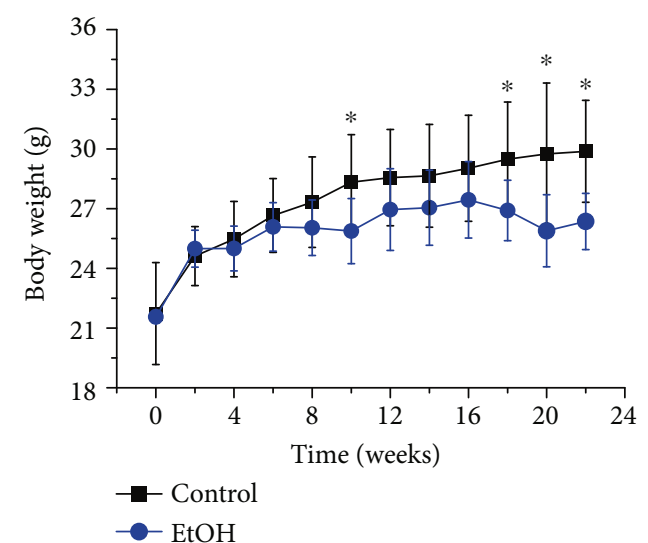

(a)

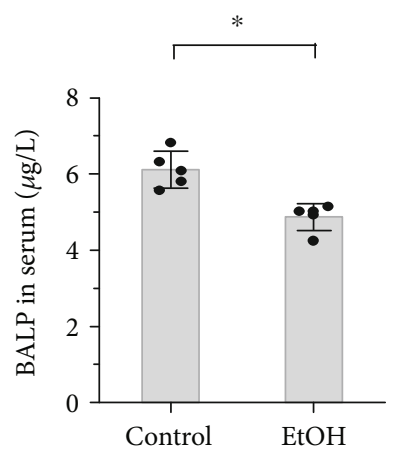

(c)

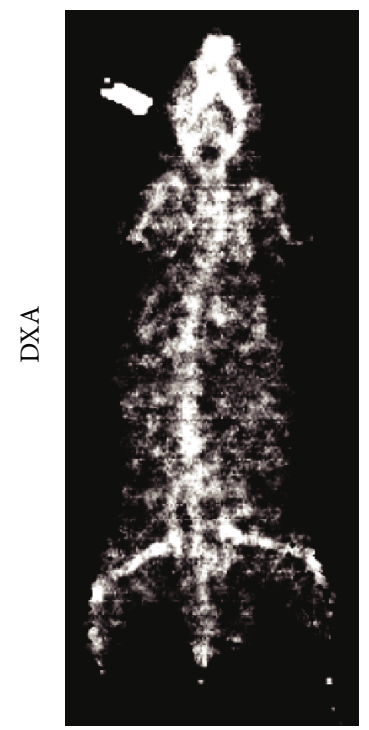

Control

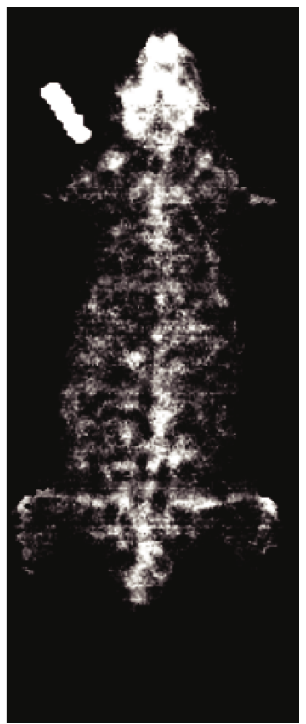

$\mathrm{EtOH}$

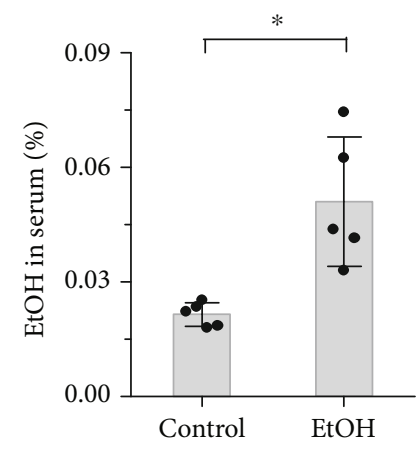

(b)

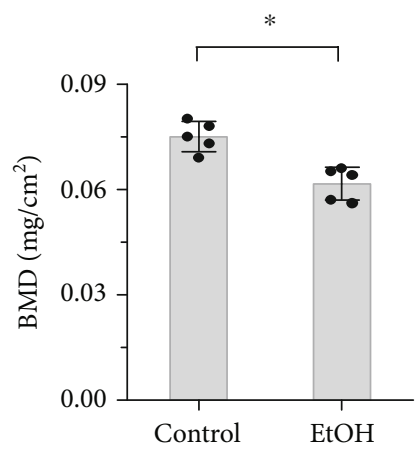

(d)

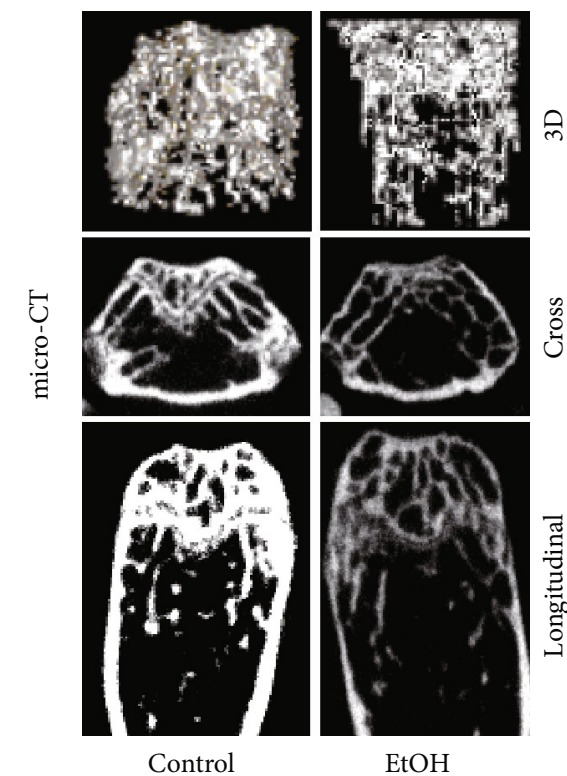

(f)

Figure 1: Continued. 

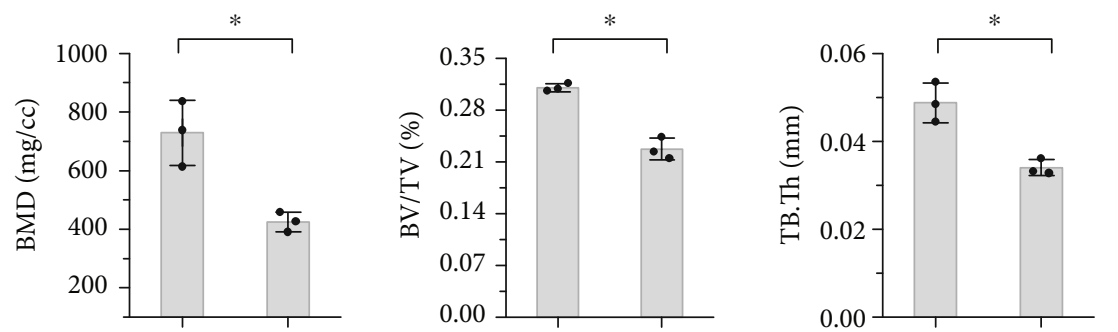

$\tilde{0}$

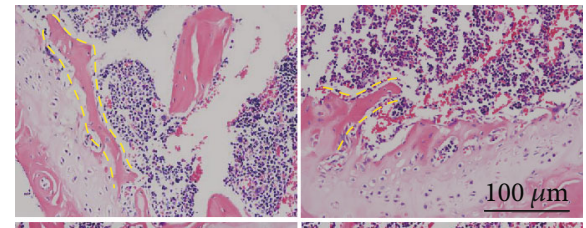

is
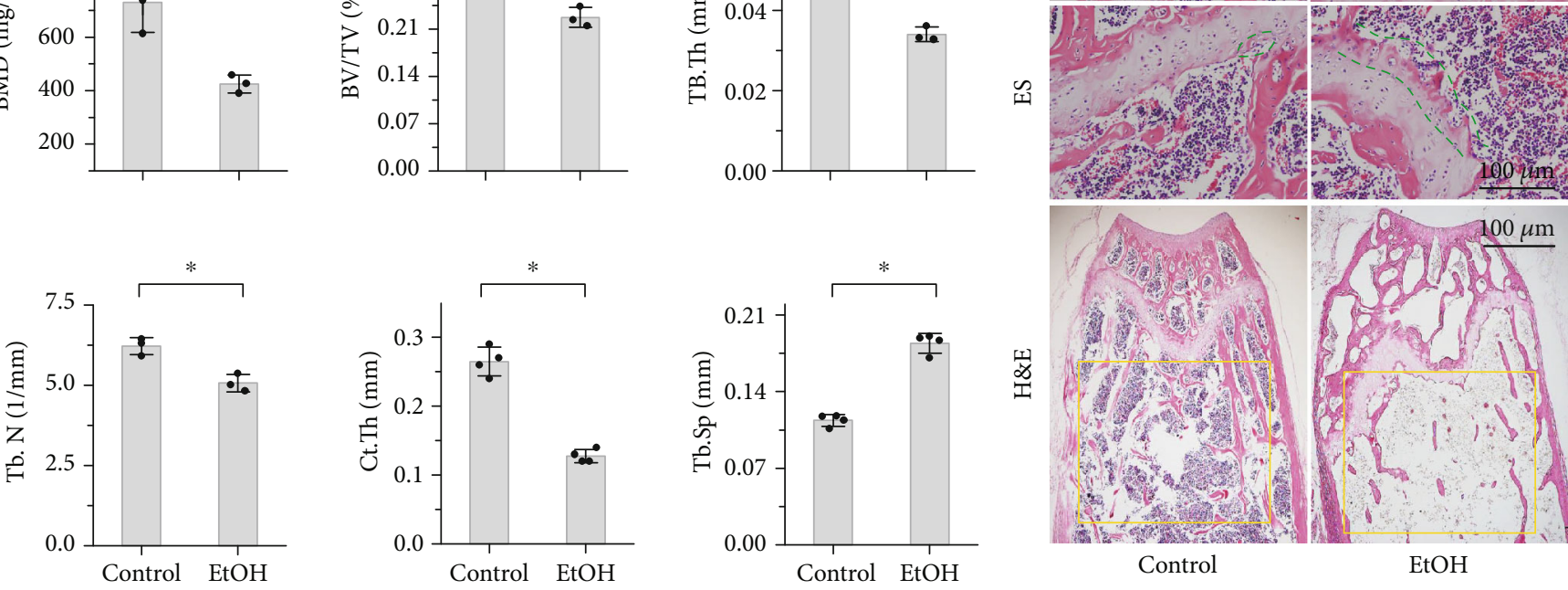

(g)
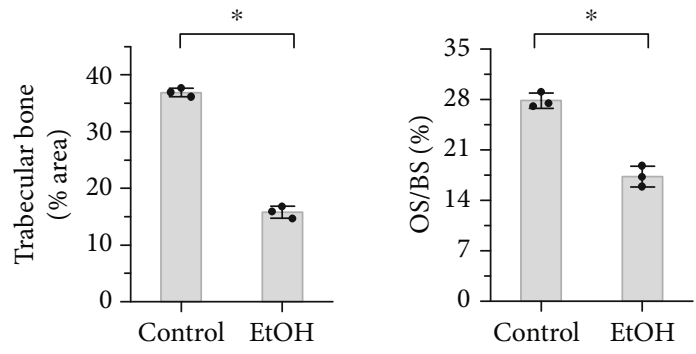

瓷
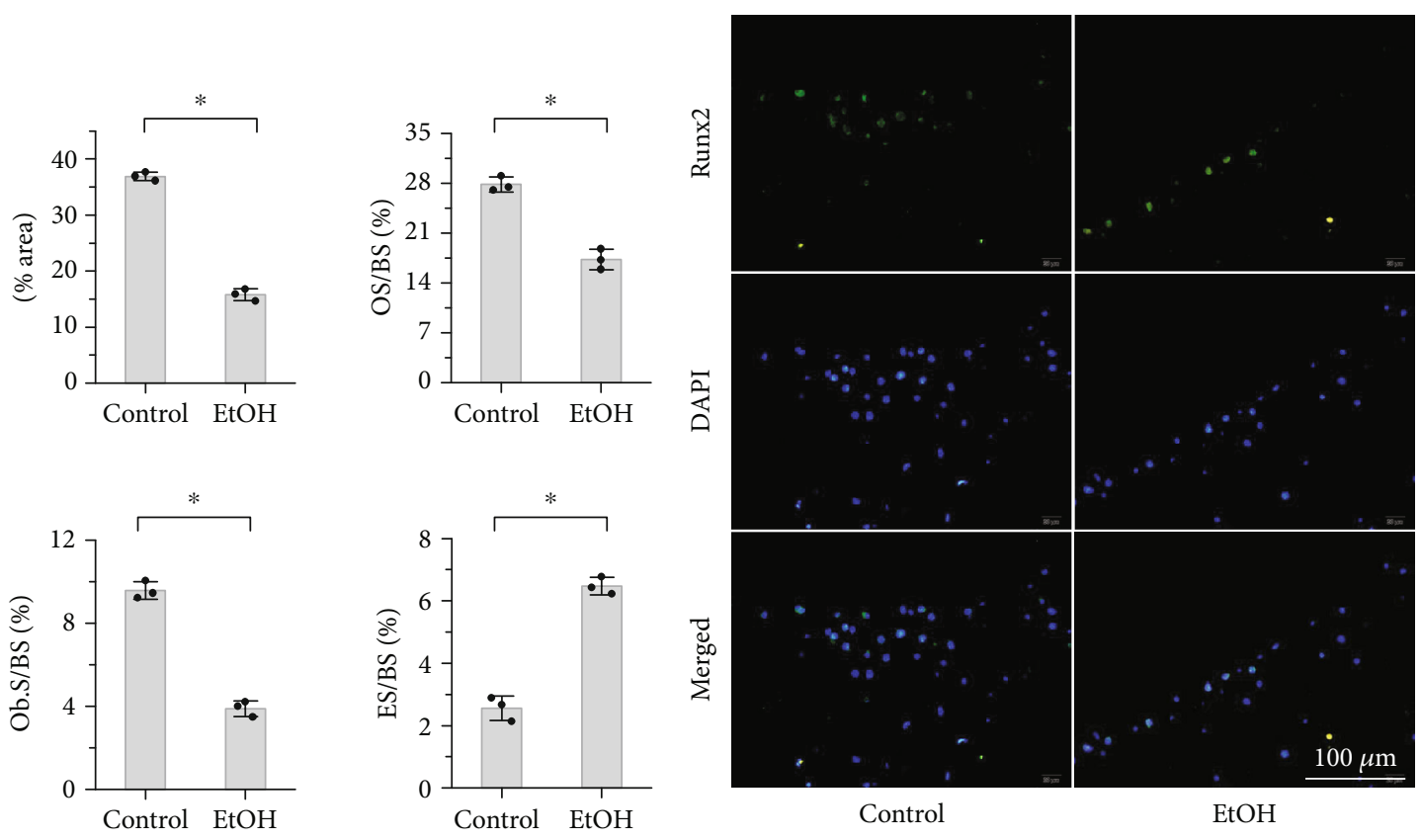

(i)

(j)

FIgURe 1: Continued. 


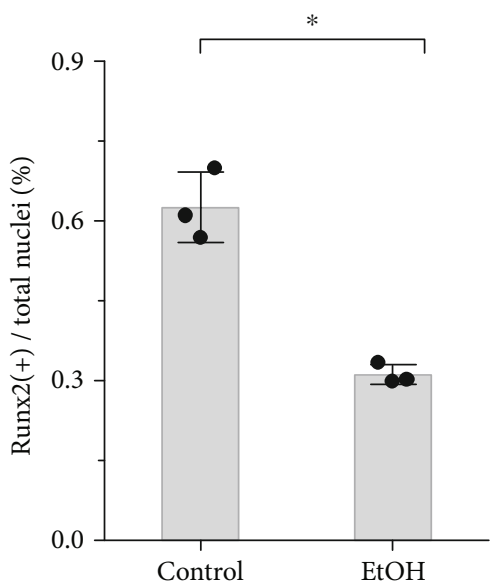

(k)

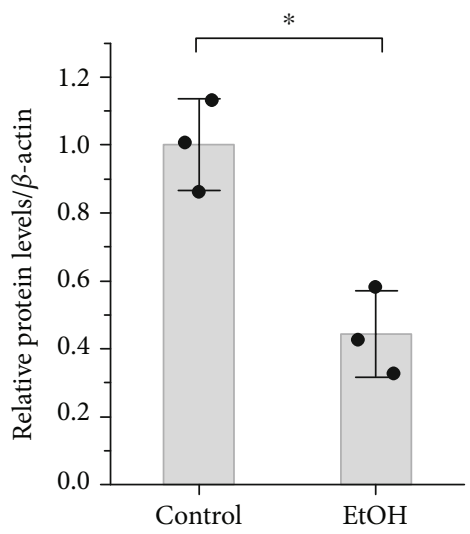

$(\mathrm{m})$
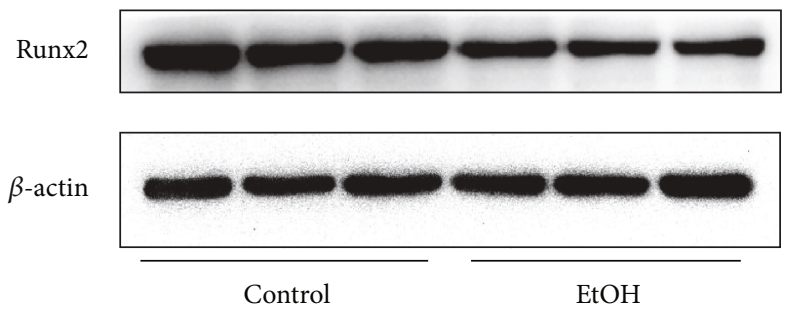

(l)

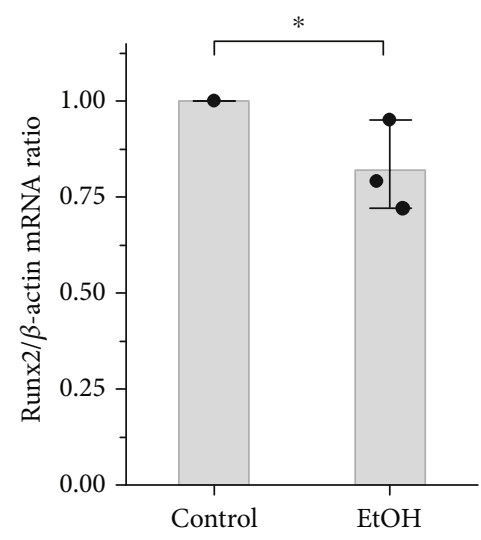

(n)

FIGURE 1: EtOH treatment leads to osteogenic differentiation reduction and osteopenia. (a) Body weight of mice for 22 weeks. (b) The serum alcohol concentration of the mice in the EtOH-treated group was twice as much as the control group when sacrificed. (c) The level of BALP in serum was decreased. (d, e) DXA analysis of mouse bilateral femurs (e) showed that chronic alcohol consumption significantly reduced the BMD of mice (d). (f, g) Micro-CT analysis of trabecular revealed that BMD, BV/TV, Tb.Th, Tb.N, and Ct.Th were markedly decreased in EtOH-treated mouse distal femurs and with the increase of Tb.Sp. (h, i) Histomorphometric analysis revealed that the distal trabecular bone structure was disordered and loose, the numbers of trabecular bone ( $h$, yellow rectangle area), the ratio of OS/BS (h, two yellow dotted lines), and Ob.S/BS were significantly decreased, and the ratio of ES/BS (h, two green dotted lines) was increased compared to untreated mice (i). Bar: $100 \mu \mathrm{m}$. $(\mathrm{j}-\mathrm{n})$ Both mRNA and protein levels of Runx2 in mouse bone tissue were significantly decreased that evaluated by immunofluorescent staining $(\mathrm{j}$, $\mathrm{k}$; bar: $100 \mu \mathrm{m})$, western bolt analysis $(\mathrm{l}, \mathrm{m})$, and RT-PCR (n). All experimental data were verified in at least three independent experiments. Error bars represent the SD from the mean values. ${ }^{*} p<0.05$. EtOH: ethyl alcohol; BALP: bone alkaline phosphatase; DXA: dual-energy X-ray absorptiometry; micro-CT: micro-computed tomography assay; BMD: bone mineral density; BV/TV: bone volume/total volume; Tb.Th: trabecular thickness; Tb.N: trabecular number; Ct.Th: cortical thickness; Tb.Sp: trabecular separation; OS: osteoid surface; ES: eroded surface; OS/BS: osteoid surface to bone surface; Ob.S/BS: osteoblast surface to bone surface; ES/BS: eroded surface to bone surface; Runx2: runt-related transcription factor 2.

Surprisingly, the expression of RIPK1 in osteoclasts had no significant difference between the alcohol intervention group and the control group with colocalization (Supplementary Figure S1c). Overall, necroptosis of osteoblasts was more obvious than osteoclasts with alcohol intervention, so our experiment mainly focused on bone formation rather than bone resorption.

To determine the type of cell death that a cell undergoes, antiactive caspase- 3 was detected. The results showed that the protein levels of cleaved caspase- 3 in mouse bone tissue and MC3T3-E1 cells were increased in the EtOH-treated group evaluated by immunofluorescent staining and western bolt analysis, but the difference was not statistically signifi- cant $(p>0.05$, Figures $2(\mathrm{~g})$ and 2(h)). Consistently, TEM analysis revealed that cells displayed typical necrotic ultrastructural changes including a disrupted plasma membrane, fragmented and vacuolated mitochondrial membranes, and overflowed cytoplasmic content after exposure to $150 \mathrm{mM}$ EtOH for $48 \mathrm{~h}$ (Figures 3(i) and 3(j)) that exhibited the typical characteristic of necroptosis rather than apoptosis. The above results suggested that apoptosis accounts for a small proportion in the occurrence of alcohol-induced osteopenia, but mainly necroptosis.

Further, to confirm the activation of necroptosis in the EtOH-treated group, we performed western blotting to semiquantitatively analyze the changes in the protein 


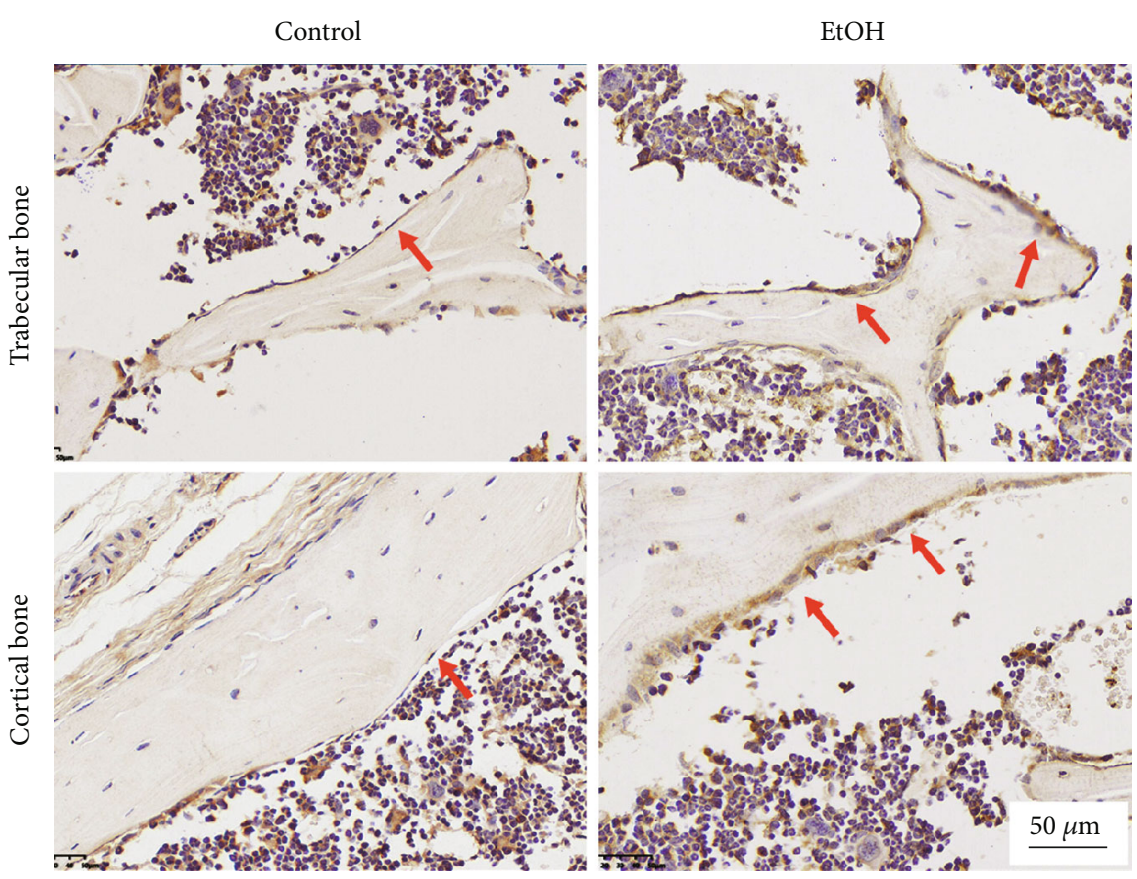

(a)

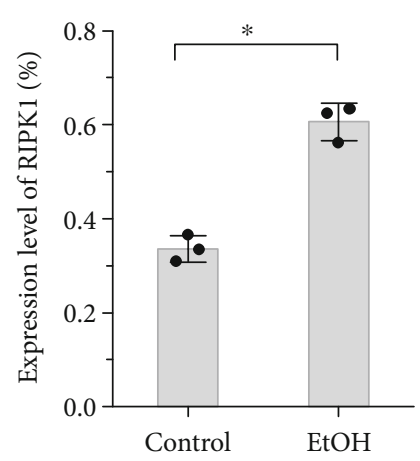

(b)

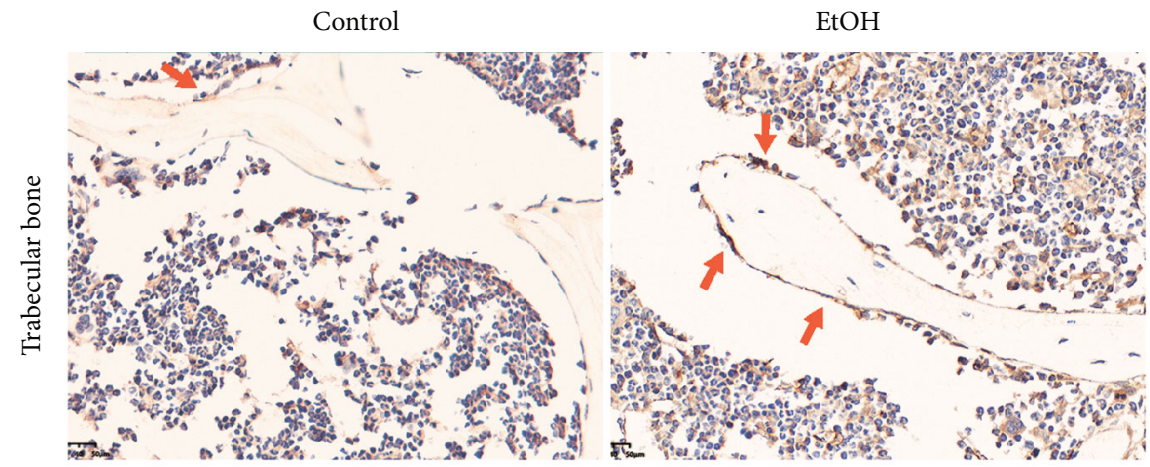

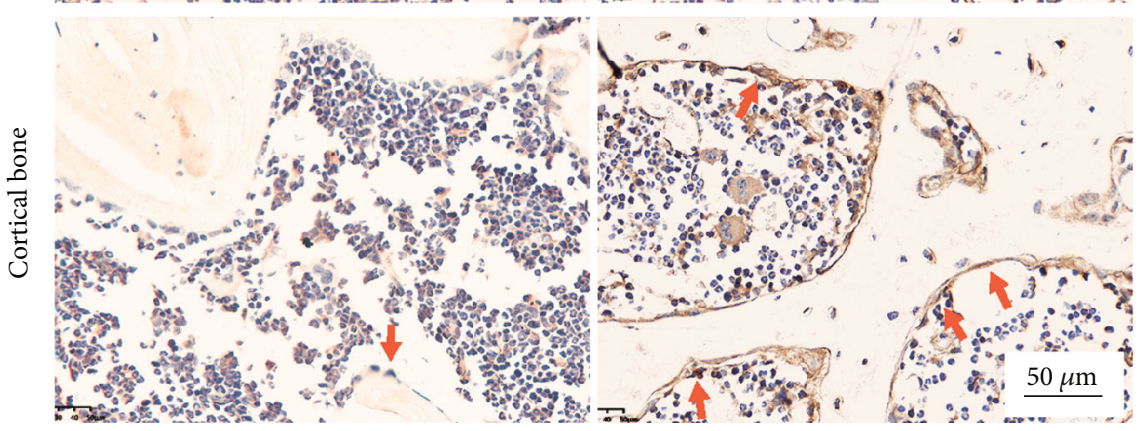

(c)

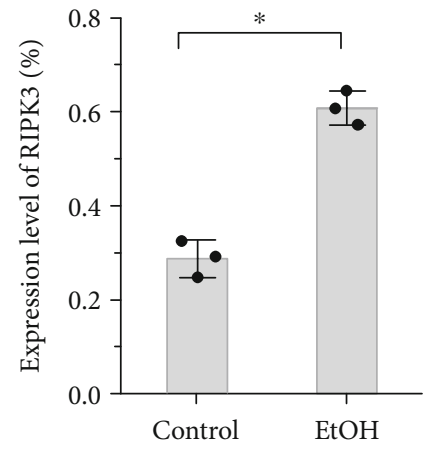

(d)

Figure 2: Continued. 


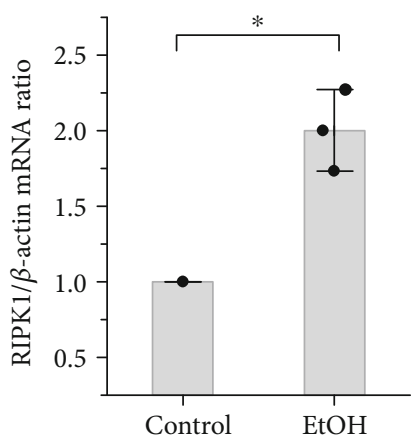

(e)

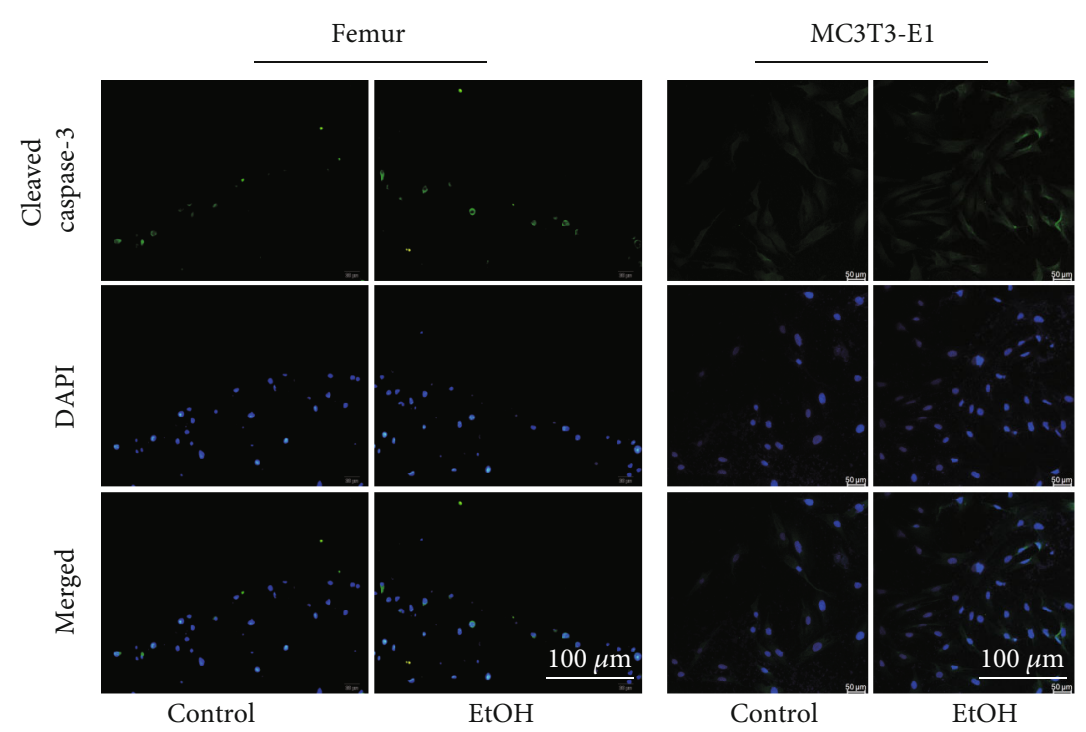

(g)

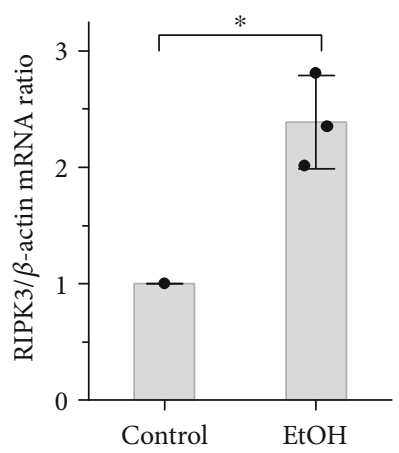

(f)
Control

Cleaved caspase-3

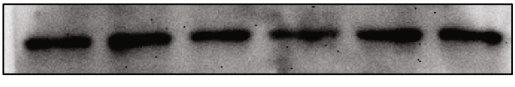

$\beta$-actin

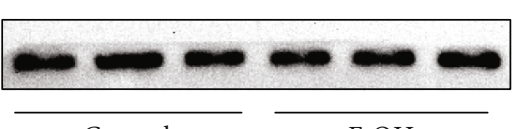

Control $\mathrm{EtOH}$

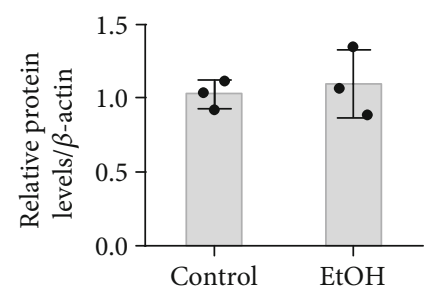

(h)

Figure 2: Continued. 


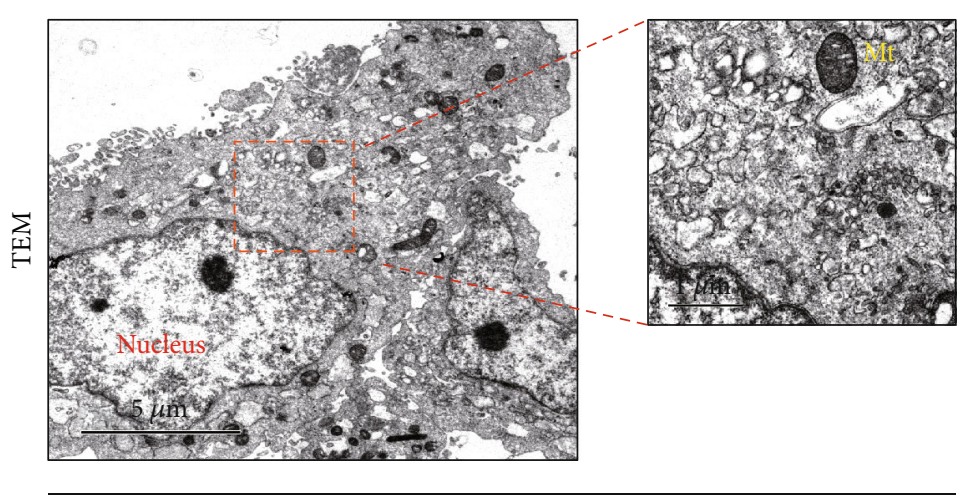

Control

(i)

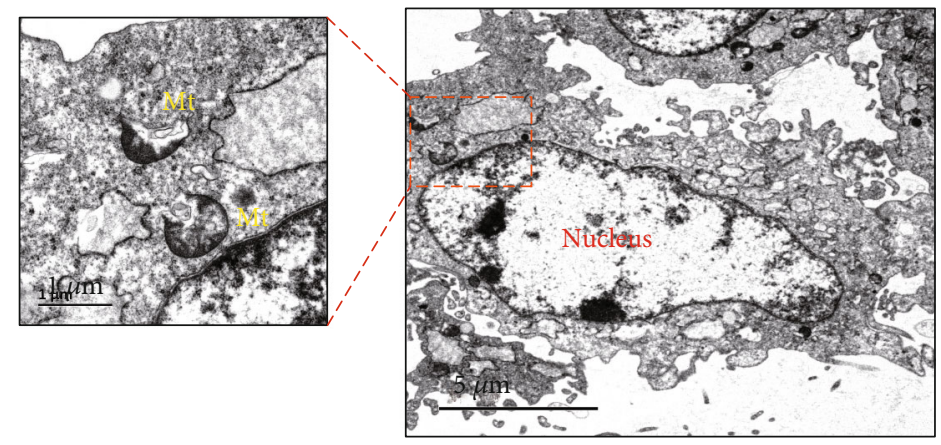

$\mathrm{EtOH}$

(j)

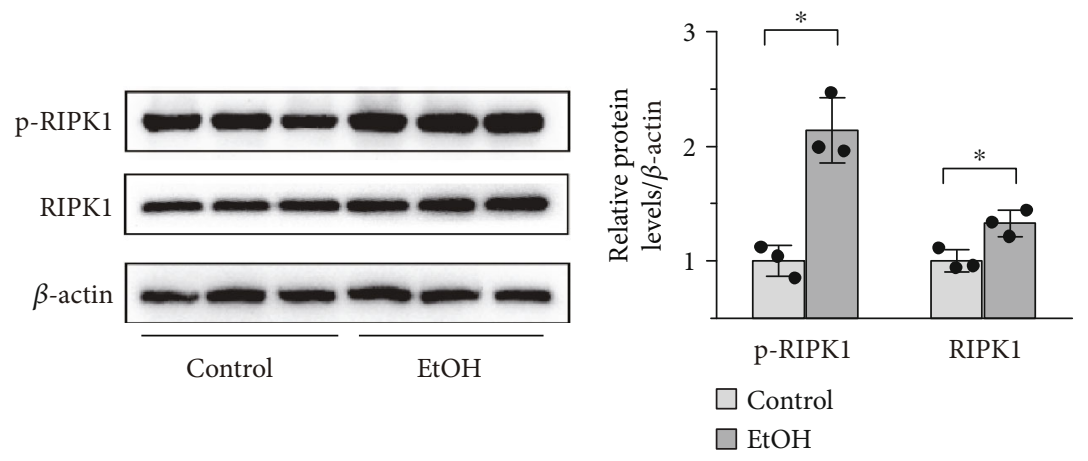

(k)

(1)

Figure 2: Continued. 


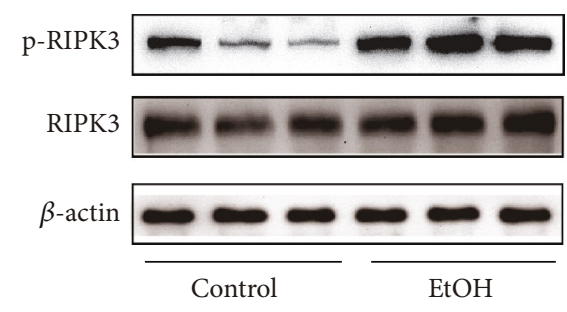

$(\mathrm{m})$

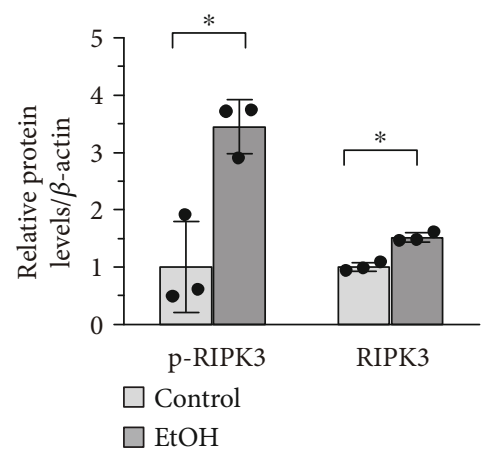

(n)

Figure 2: EtOH treatment leads to osteoblast necroptosis in vivo. (a-d) Immunohistochemistry (IHC) staining to evaluate the differences in RIPK1 and RIPK3 expression and identify osteoblasts according to the morphology and distribution in bone tissue, finally located the expression of RIPK1 and RIPK3 on osteoblasts. The number of RIPK1-positive (a, b) and RIPK3-positive (c, d) osteoblasts markedly increased in femurs with EtOH treatment. The red arrow indicates the positive expression in osteoblasts. Bar: $50 \mu \mathrm{m}$. (e, f) Real-time PCR showed upregulated in the mRNA expression of RIPK1 and RIPK3 with EtOH treatment. Immunofluorescent staining of femurs and MC3T3-E1 cells (g) and western blot of mouse bone tissue (h) showed that the protein levels of cleaved caspase-3 were increased in the EtOH-treated group, but the difference was not statistically significant $(p>0.05)$. Bar: $100 \mu \mathrm{m}$. TEM analysis revealed that cells exhibited normal nuclear and cytoplasmic morphology in the control group (i) and displayed typical necrotic ultrastructural changes including a disrupted plasma membrane, fragmented and vacuolated mitochondrial membranes (yellow mark), and overflowed cytoplasmic content after exposure to $150 \mathrm{mM} \mathrm{EtOH}$ for $48 \mathrm{~h}$ (j). Bar: $5 \mu \mathrm{m}$ and $1 \mu \mathrm{m}$, respectively. Western blotting showed upregulated in the protein expression of RIPK1 and p-RIPK1 $(\mathrm{k}, \mathrm{l})$ and RIPK3 and p-RIPK3 $(\mathrm{m}, \mathrm{n})$ with EtOH treatment in vivo. Error bars represent the SD from the mean values. ${ }^{*} p<0.05$. RIPK1: receptor-interacting serine/threonine kinases 1 ; RIPK3: receptor-interacting serine/threonine kinases 3; TEM: transmission electron microscopy; Mt: mitochondrion.

expression levels. The results showed upregulated expression of RIPK1 and p-RIPK1 (Figures 2(k) and 2(l)) and RIPK3 and p-RIPK3 (Figures $2(\mathrm{~m})$ and $2(\mathrm{n})$ ) with EtOH treatment in vivo, indicating that chronic heavy alcohol-induced bone loss in mice was associated with osteoblast necroptosis activation.

\subsection{EtOH Induces Osteoblast Necroptosis by Activating the} RIPK1/RIPK3/MLKL Signaling. To investigate the mechanism of EtOH-induced osteoblast necroptosis, we added $150 \mathrm{mM}$ EtOH to BMMSCs, which were identified by flow cytometry analysis (Supplementary Figure S2a) and then induced to differentiate into osteoblasts that were evaluated by alkaline phosphatase (ALP) staining (Supplementary Figure S2b) and alizarin red staining (Supplementary Figure S2c), for $48 \mathrm{~h}$ using the CCK-8 assay (Supplementary Figure S3a, b). Quantitative western blotting showed that EtOH treatment elevated the expression of RIPK1 and p-RIPK1 (Figures 3(a) and 3(b)), RIPK3 and p-RIPK3 (Figures 3(c) and 3(d)), and p-MLKL (Figures 3(e) and 3(f)), while the expression of caspase-8 was not significantly influenced (Figures $3(\mathrm{~g})$ and $3(\mathrm{~h})$ ). Double-labeled immunofluorescence staining confirmed that MC3T3-E1 significantly coexpressed the necroptosis markers RIPK1 and RIPK3 after EtOH treatment (Figure 3(i)). Confocal microscopy further proved that $\mathrm{p}$ MLKL aggregated on the cell membrane, leading to perforation and destruction of the integrity of proliferating MC3T3-E1 (Figure 3(j)). Therefore, we concluded that alcohol induced osteoblast necroptosis by activating the RIPK1/RIPK3/MLKL signaling.
3.4. Necrostatin-1 Treatment Ameliorates Osteopenia and Osteogenic Differentiation Reduction in EtOH-Treated Mice. Next, to examine whether necrostatin-1 treatment ameliorated osteopenia in $\mathrm{EtOH}$-treated mice, we treated the mice with $1.65 \mathrm{mg} / \mathrm{kg} / \mathrm{d}$ of $\mathrm{Nec}-1$ for 22 weeks and BMMSCs with $50 \mu \mathrm{M}$ of Nec-1 for $48 \mathrm{~h}$, respectively (Supplementary Figure S3c, d). We found that Nec-1 treatment ameliorated EtOH-induced low BMD, trabecular BV/TV, Tb.Th, Tb.N, and Ct.Th in the distal femurs, as assessed by DXA, micro-CT cross sections, and 3D reconstructions, respectively (Figures 4(a)-4(d)). Further, H\&E staining revealed that $\mathrm{Nec}-1$ treatment inhibited the reduction in trabecular bone volume and number, facilitated partial recovery of the loose trabecular structure, and increased the ratio of OS/BS and Ob.S/BS compared to EtOH-treated mice (Figures 4(e) and 4(f)). Moreover, Nec-1 treatment significantly increased bone formation, as indicated by the increase in alkaline phosphatase activity and the formation of mineralized nodules in osteoblasts (Figure $4(\mathrm{~g})$ ) and serum ALP activity in mice (Figure 4(h)). Further, immunofluorescence staining (Figures 5(a) and 5(b)), qPCR (Figure 5(c)), and western blot analysis both in vivo (Figures 5(d) and 5(e)) and in vitro (Figures 5(f) and 5(g)) showed that the expression level of Runx2 was downregulated after EtOH treatment; notably, necrostatin1 treatment upregulated these expression levels.

3.5. Necrostatin-1 and NAC Treatment Ameliorates Osteopenia by Inhibiting the RIPK1/RIPK3/MLKL Signaling. We further explored whether necrostatin-1 treatment could inhibit RIPK1 kinase and investigated its effects 


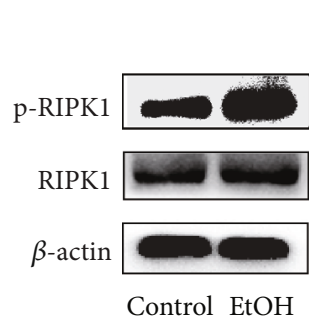

(a)

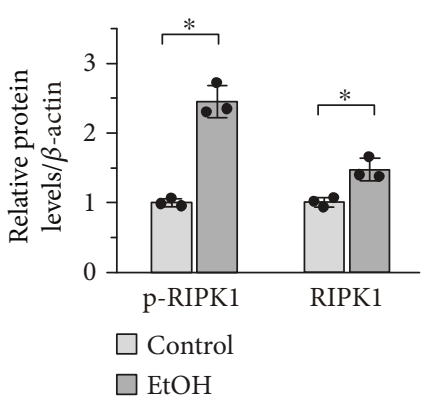

(b)

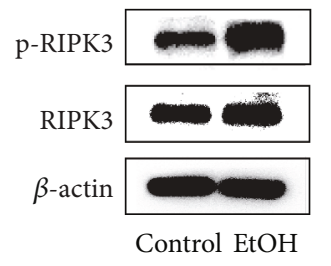

(c)

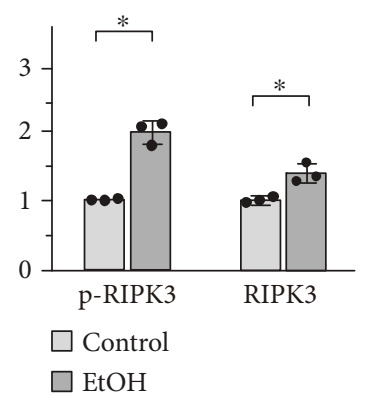

(d)

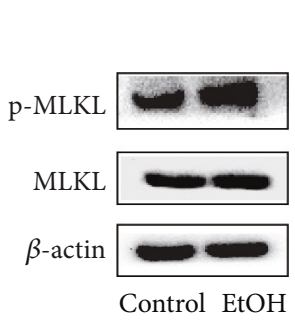

(e)

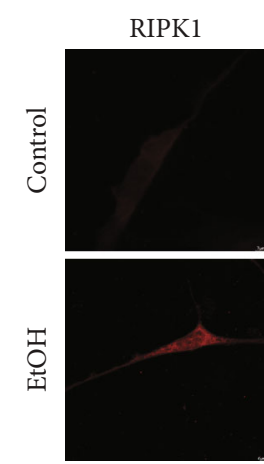

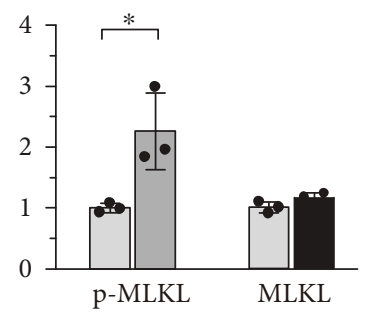

$\square$ Control

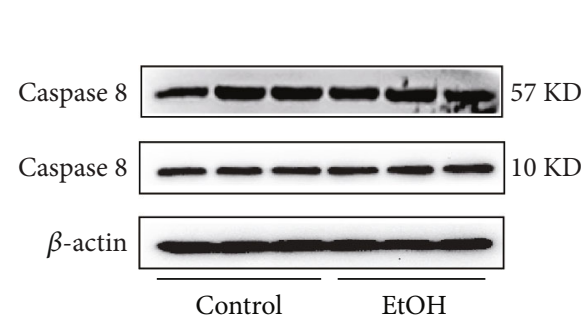

(g)
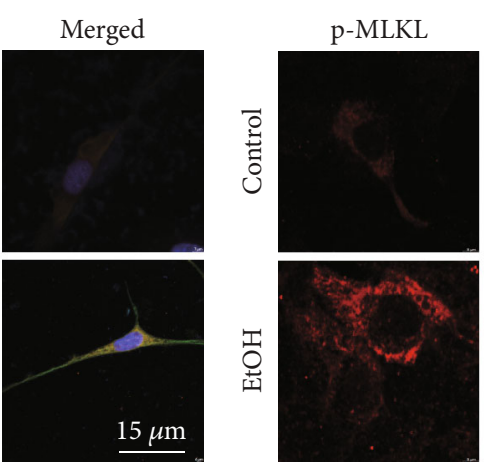

(i)

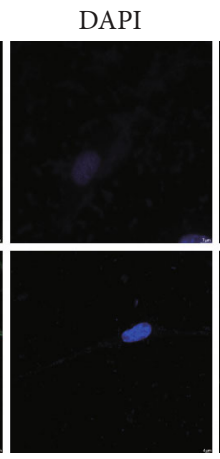

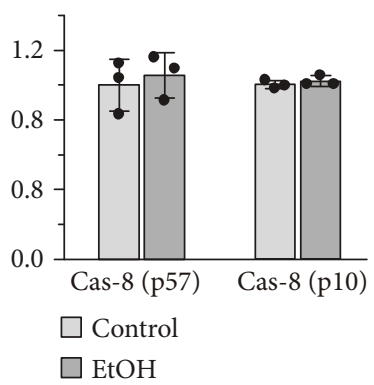

(h)

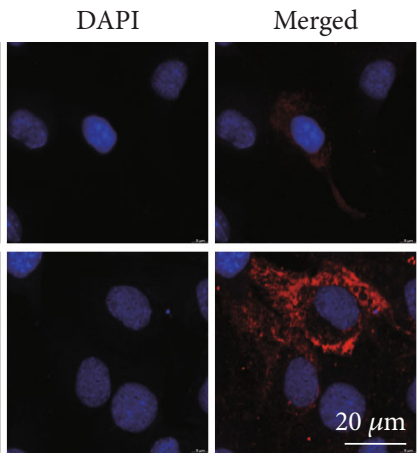

(j)

FIgURE 3: EtOH induces osteoblast necroptosis by activating the RIPK1/RIPK3/MLKL signaling in vitro. Western blot showed that the expressions of RIPK1 and p-RIPK1 (a, b), RIPK3 and p-RIPK3 (c, d), and p-MLKL (e, f) on MC3T3-E1 cells were elevated in the EtOH treatment group, while the expression of caspase- 8 was not significantly influenced $(\mathrm{g}, \mathrm{h})$. Double-labeled immunofluorescence staining confirmed that necroptosis makers RIPK1 and RIPK3 coexpressed in the EtOH-treated MC3T3-E1 cells (i). Bar: $15 \mu \mathrm{m}$. And p-MLKL aggregated on the cell membrane, leading to perforation and destruction of the integrity of proliferating MC3T3-E1 osteoblasts (j). Bar: $20 \mu \mathrm{m}$. Error bars represent the SD from the mean values. ${ }^{*} p<0.05$. p-MLKL: phosphorylated mixed lineage kinase domain-like protein.

on the downstream signaling pathway of RIPK1 with EtOH intervention. Western blot analysis showed that Nec-1 treatment lowered the elevated levels of p-RIPK1, p-RIPK3, and p-MLKL both in vivo (Figure 6(a)) and in vitro (Figure 6(b)). These results were confirmed by RT-PCR analysis, as shown in Supplementary Figure S4a-c. In the alcohol mouse model, the increased expression of RIPK1 in osteoblasts was significantly inhibited by Nec-1 treatment, as located and observed by immunohistochemistry staining (Figures 6(c) and 6(d)). In addition, confocal laser scanning microscopy revealed that $\mathrm{Nec}-1$ treatment alleviated the increase of RIPK1- and RIPK3-positive MC3T3-E1 in vitro (Figure 6(e)). These results suggest that necrostatin-1 treatment ameliorates osteopenia in EtOH-treated mice by inhibiting the RIPK1/RIPK3/MLKL signaling.
Oxidative stress is known as one trigger for alcoholinduced osteopenia, and increased levels of ROS may directly precede the production of inflammatory cytokines in the bone marrow and contribute to decreased bone formation [16]. However, whether ROS could increase osteoblast necroptosis in alcohol-induced osteopenia remains unclear. Therefore, we measured the effect of alcohol intervention on the intracellular ROS levels by flow cytometry, and our results supported that high dose of EtOH consumption increased the intracellular ROS levels compared with the control group, and Nec-1 directly inhibited the elevated ROS (Figure 6(f)). Several necroptotic stimuli have been proposed to induce necroptosis, and ROS are essential factors [46]. Next, we explored whether ROS are important for EtOH-induced necroptosis in osteoblastic cells, using the 


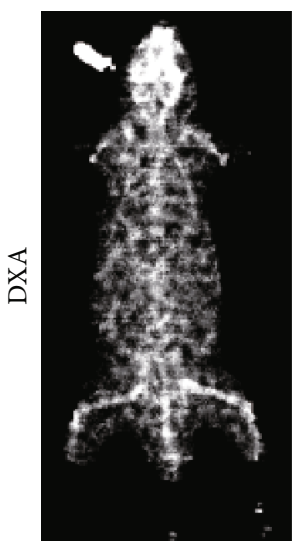

Control

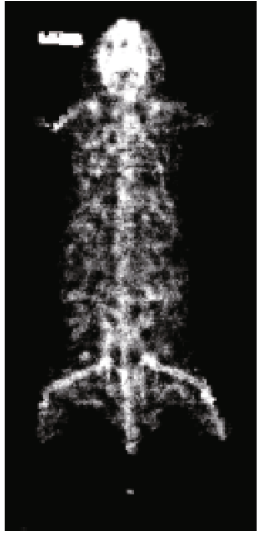

Nec-1

(a)
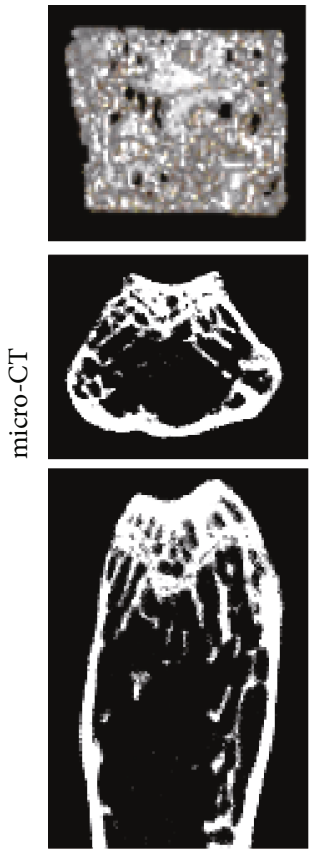

Control

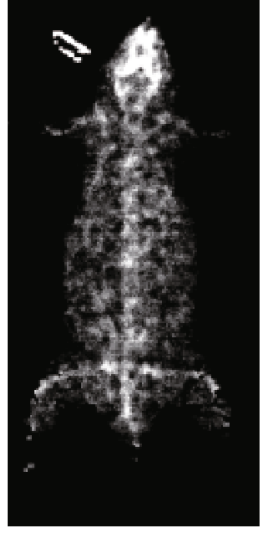

$\mathrm{EtOH}$
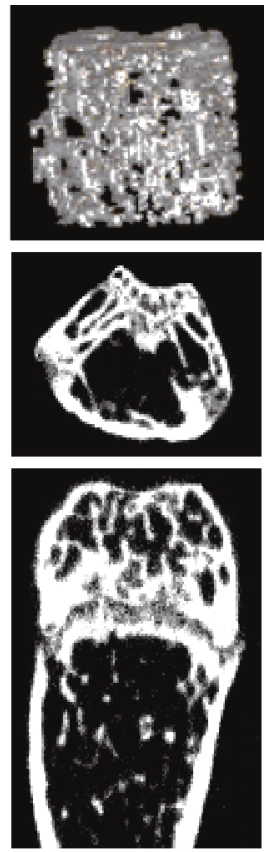

$\mathrm{Nec}-1$

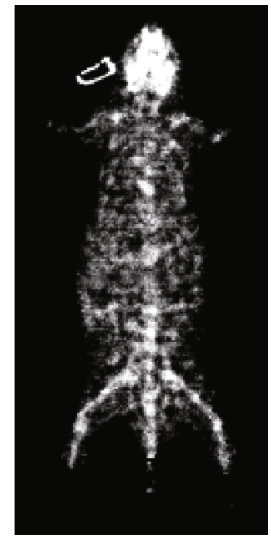

$\mathrm{EtOH}+\mathrm{Nec}-1$
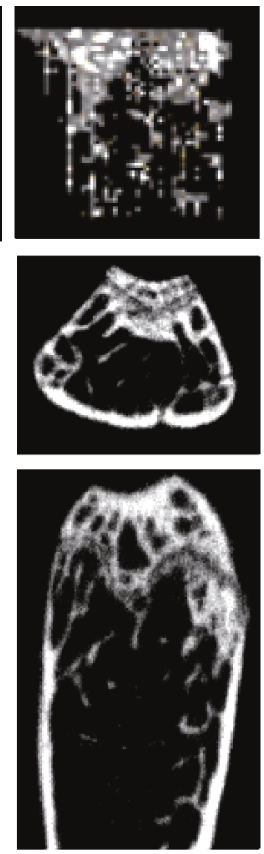

$\mathrm{EtOH}$

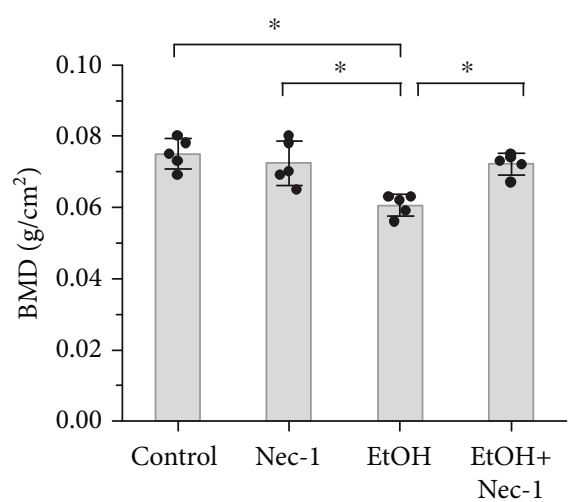

(b)
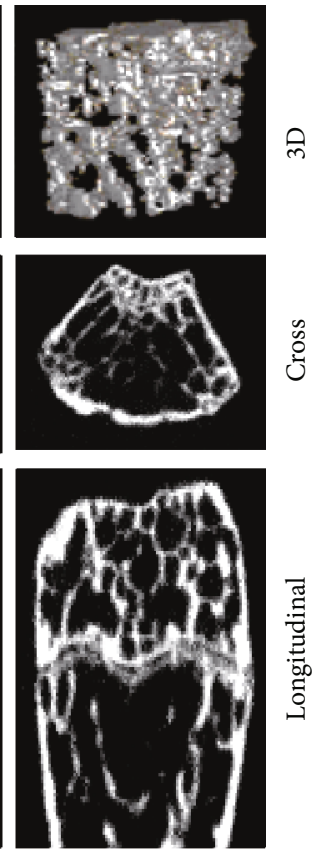

$\mathrm{EtOH}+\mathrm{Nec}-1$

(c)

FIgure 4: Continued. 

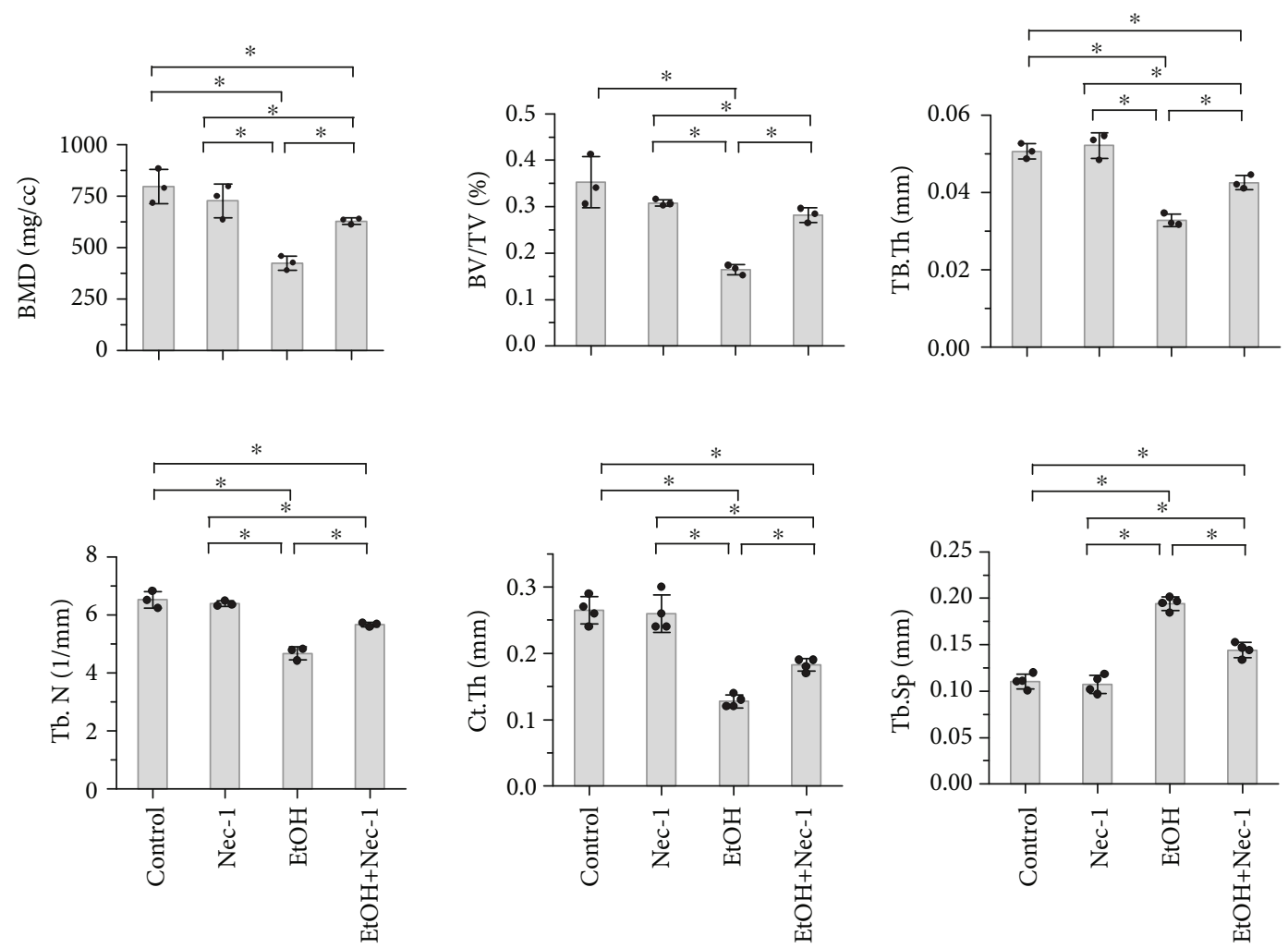

(d)

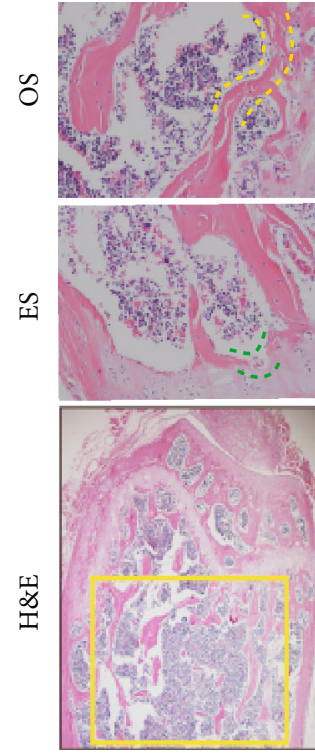

Control

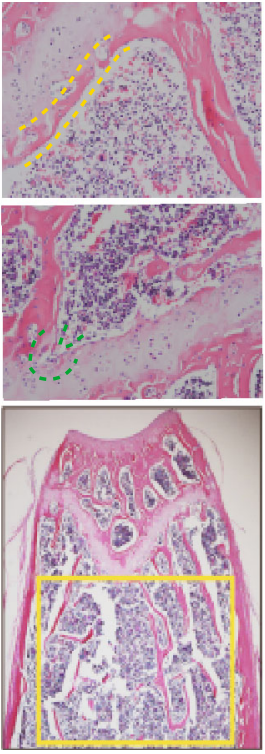

Nec-1

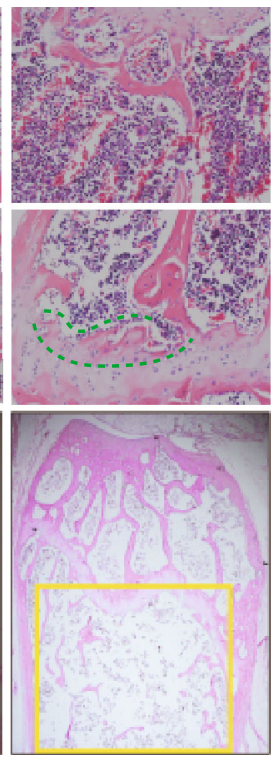

$\mathrm{EtOH}$

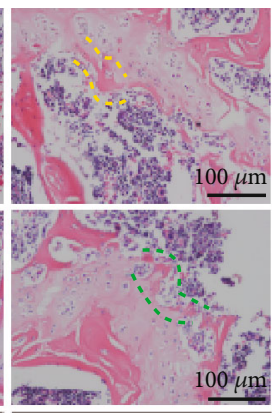

(e)

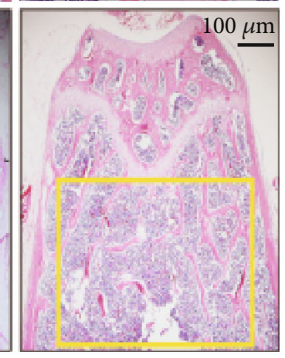

$\mathrm{EtOH}+\mathrm{Nec}-1$

Figure 4: Continued. 

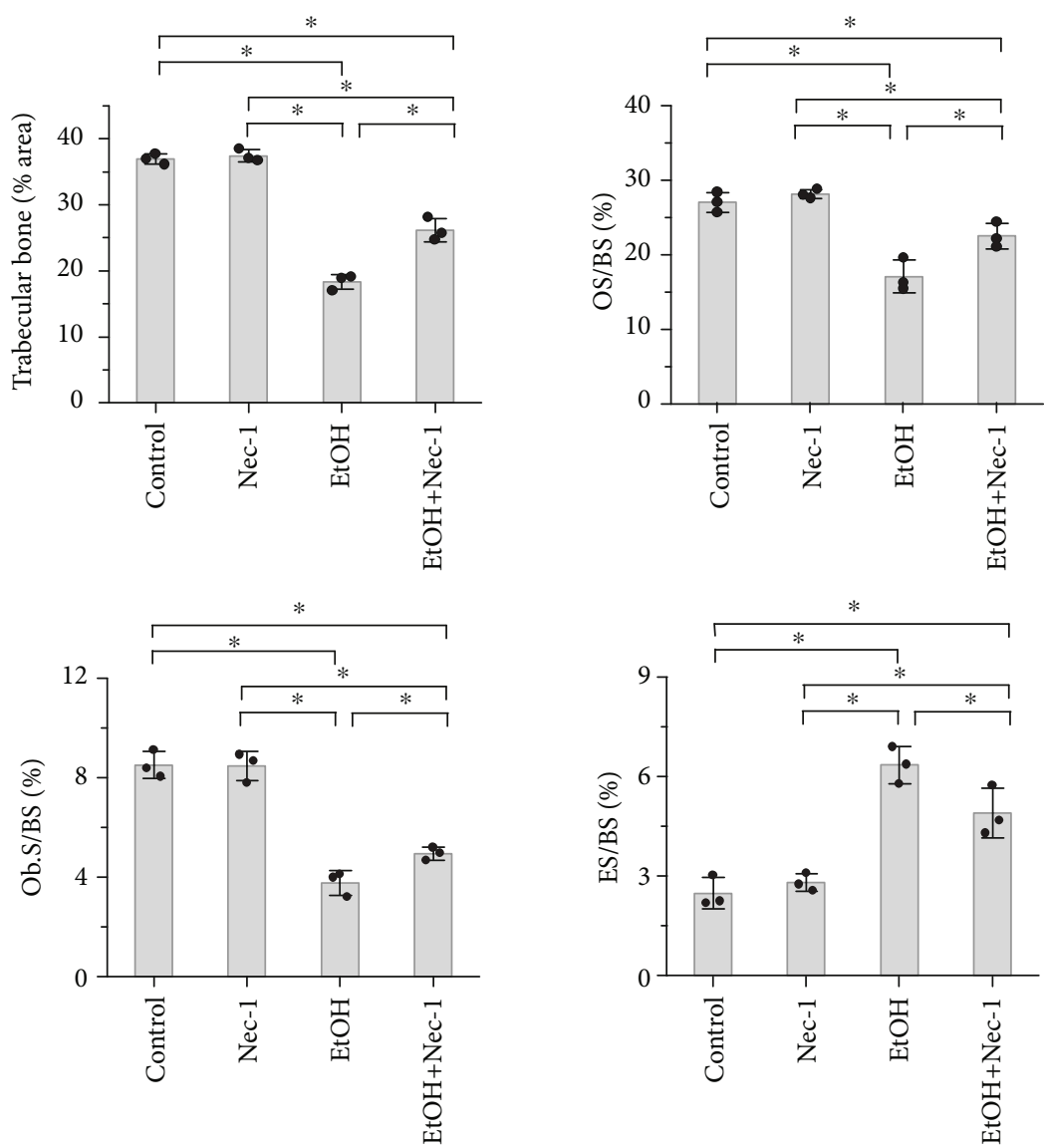

(f)

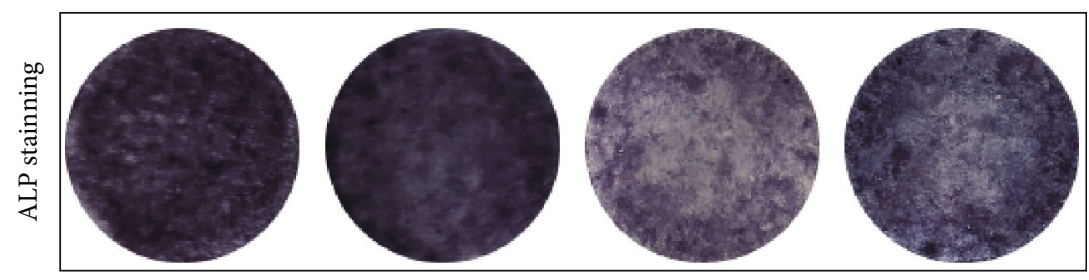

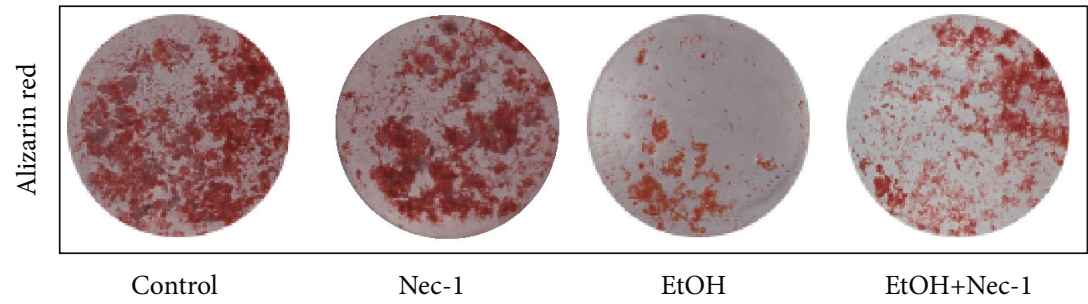

(g)

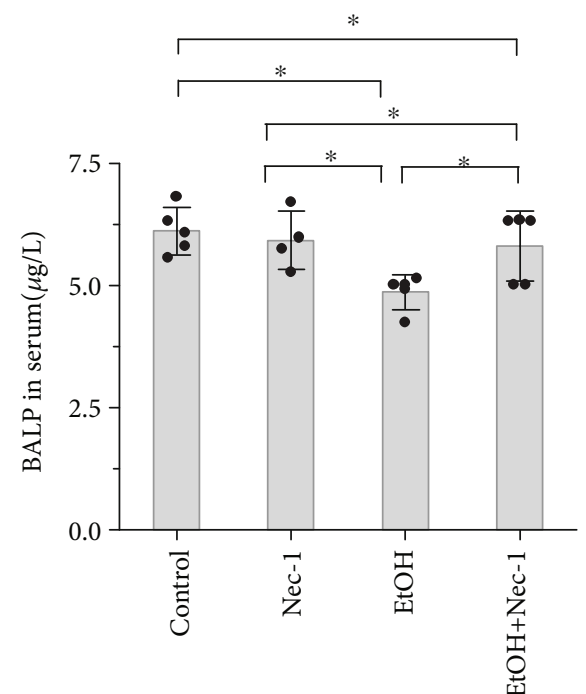

(h)

FIgURE 4: Necrostatin-1 treatment ameliorates osteopenia in EtOH-treated mice. Nec-1 treatment ameliorated EtOH-induced low BMD in femurs (a-d) and BV/TV, Tb.Th, Tb.N, and Ct.Th in the distal femurs, as assessed by DXA (a, b) and axial micro-CT cross sections and 3D reconstructions (c, d), respectively. $\mathrm{H} \& \mathrm{E}$ stained sections revealed that Nec-1 treatment inhibited the reduction in trabecular bone volume and number and facilitated partial recovery of the destroyed trabecular structure (e, yellow rectangle area), increased the ratio of OS/BS (e, two yellow dotted lines) and Ob.S/BS, and decreased the ratio of ES/BS (e, two green dotted lines) compared to EtOH-treated mice (f) Bar: $100 \mu \mathrm{m}$. Nec-1 treatment significantly increased bone formation, as indicated by the increase in alkaline phosphatase activity (ALP staining) and the formation of mineralized nodules (alizarin red staining) in osteoblasts (g) and serum alkaline phosphatase activity in mice (h). Error bars represent the SD from the mean values. ${ }^{*} p<0.05$. Nec-1: necrostatin-1. 


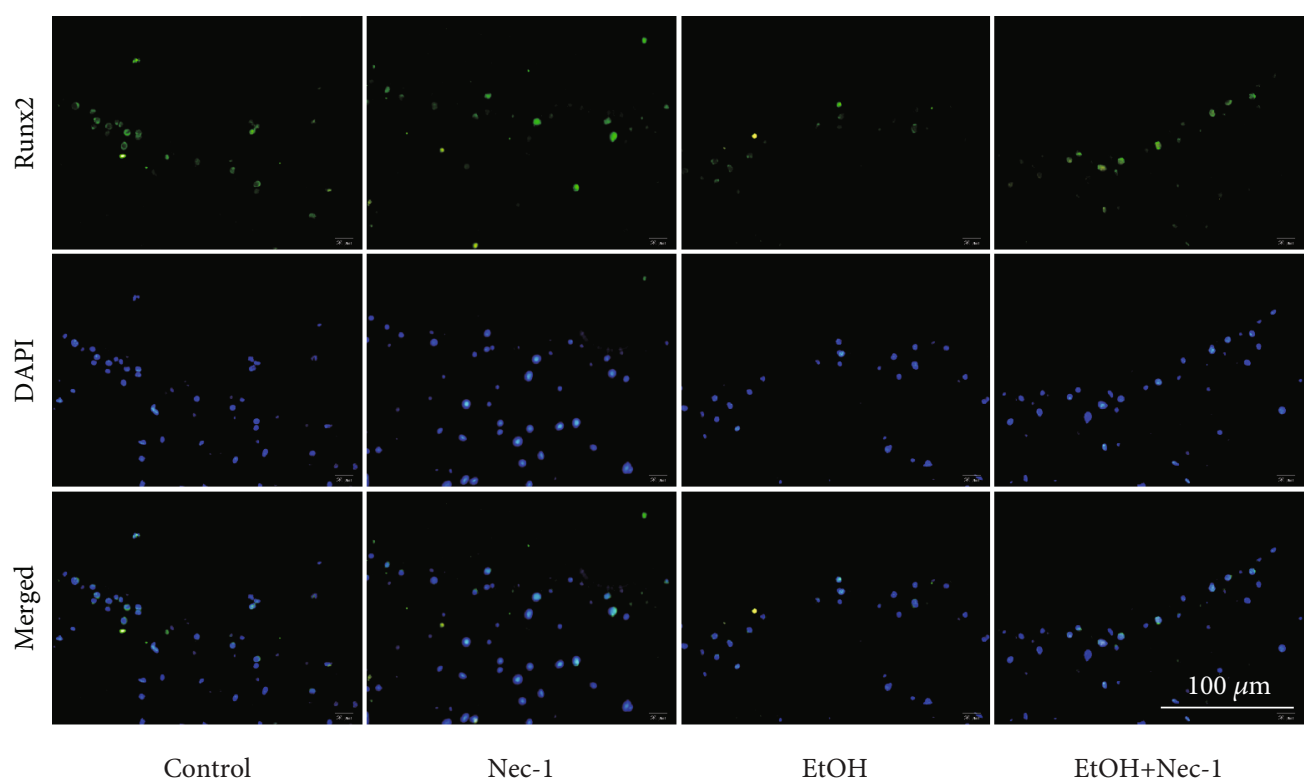

(a)

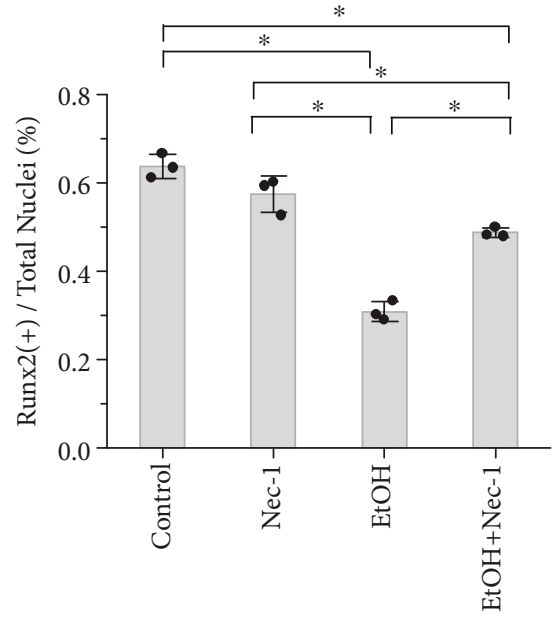

(b)

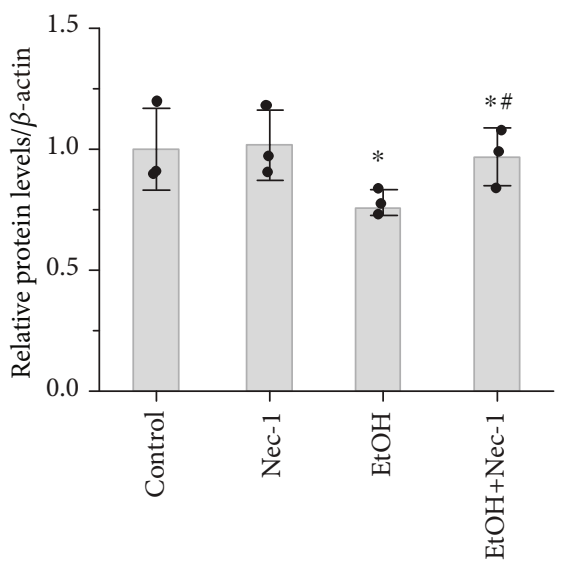

(e)

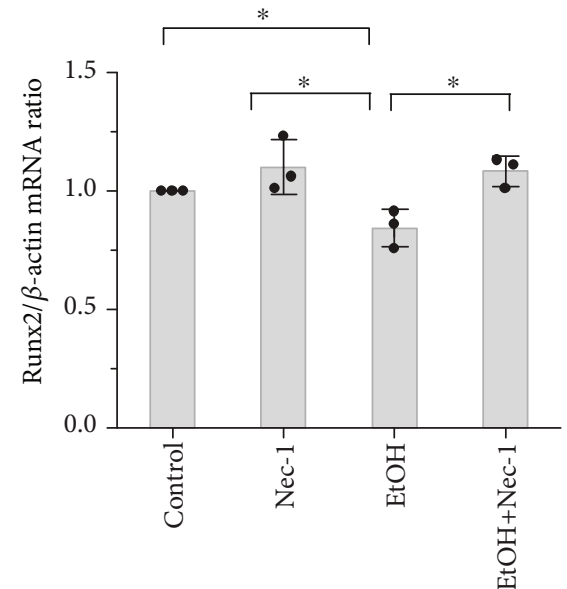

(c)

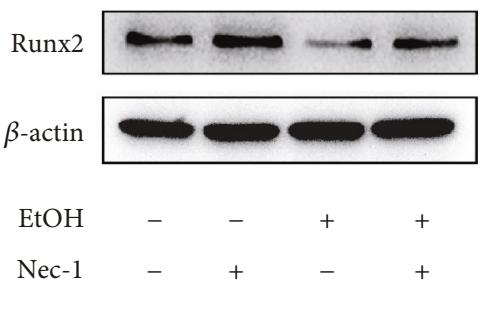

(f)

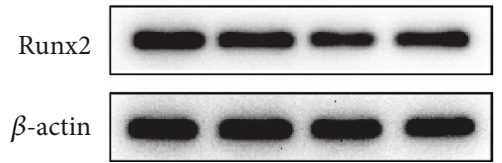

$\mathrm{EtOH}$

Nec-1

(d)

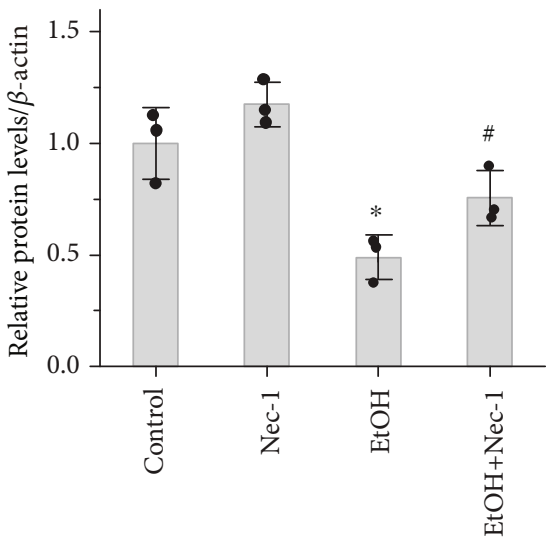

(g)

FIGURE 5: Necrostatin-1 treatment ameliorates osteogenic differentiation reduction. Immunofluorescence staining (a, b; bar: $100 \mu \mathrm{m}), \mathrm{RT}$ PCR (c), and western blot analysis both in vivo (d, e) and in vitro (f, g) showed that the expression level of Runx2 was downregulated after EtOH treatment; however, necrostatin-1 treatment upregulated the expression level. (a-e) Data from mice. (f, g) Osteoblasts. Error bars represent the SD from the mean values. ${ }^{*} p<0.05$ compared with the control group. ${ }^{\#} p<0.05$ compared with the EtOH-treated group. 


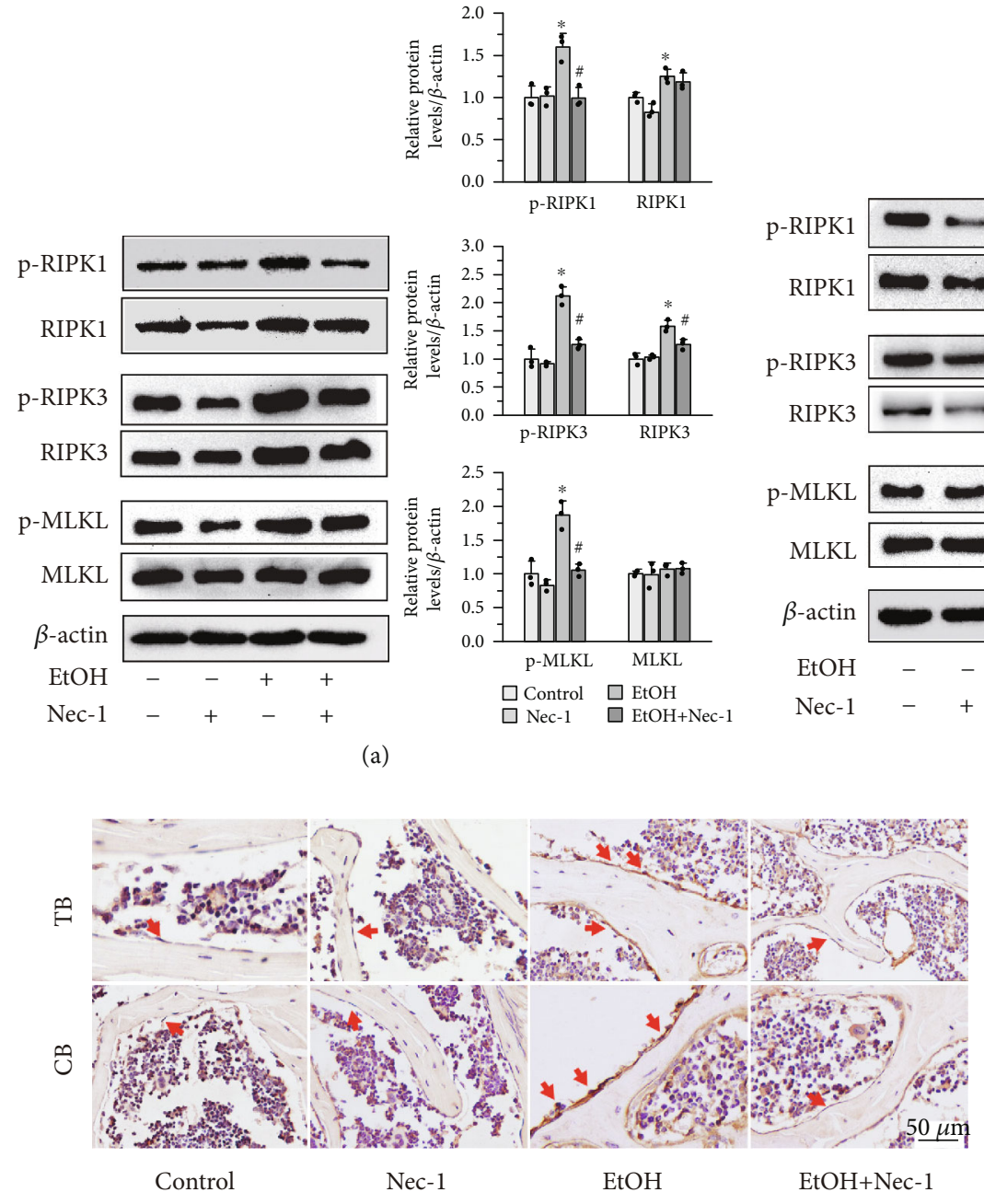

(c)
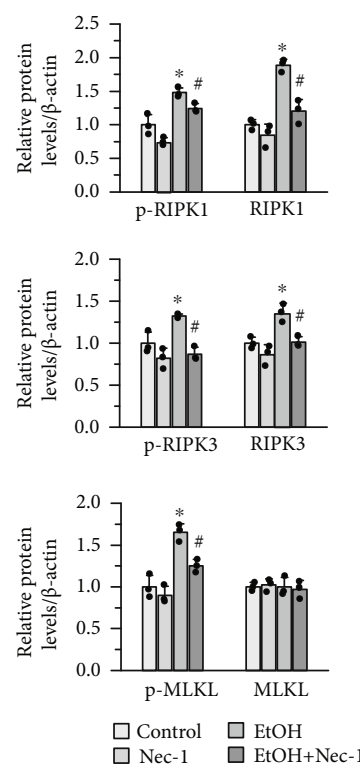

(b)

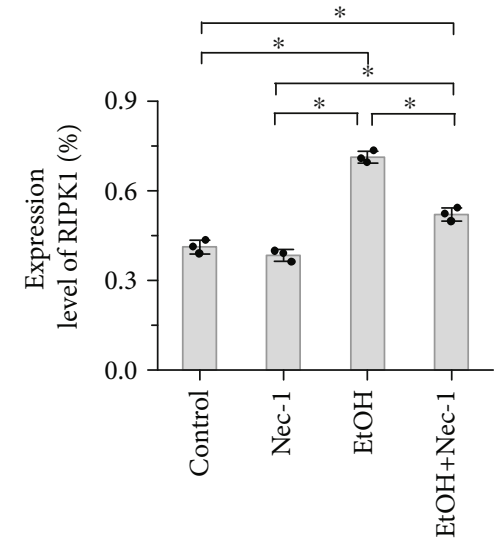

(d)

Figure 6: Continued. 


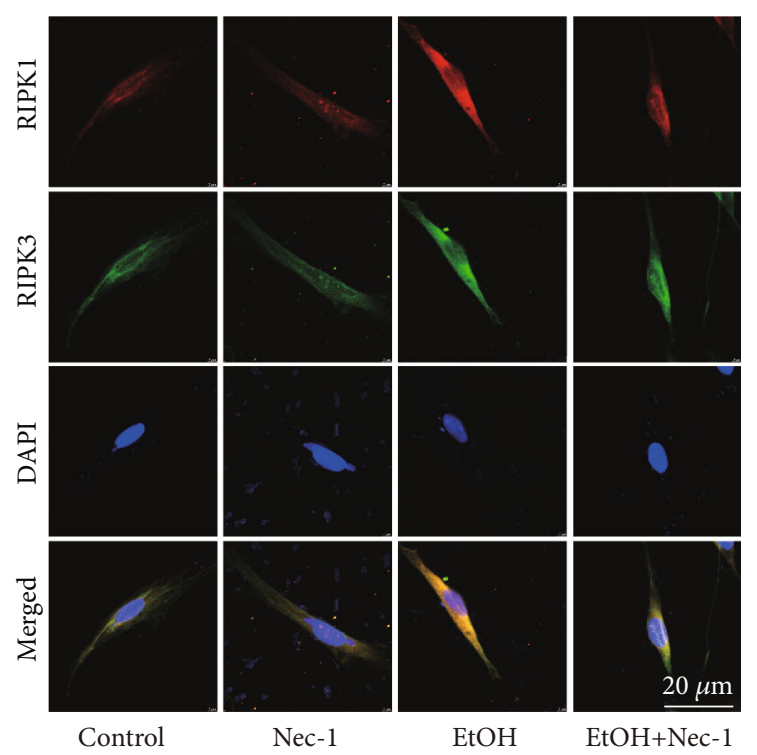

(e)

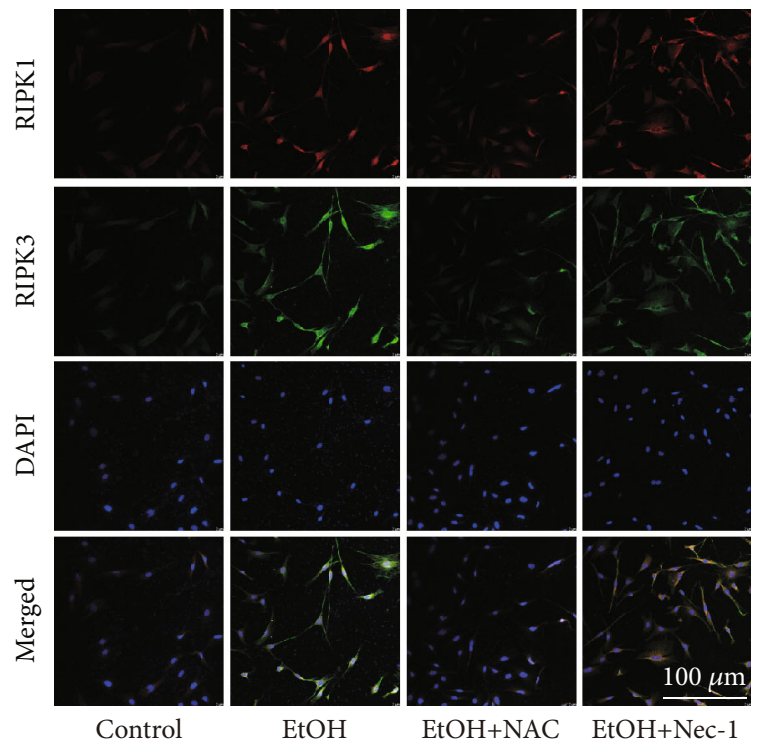

(g)

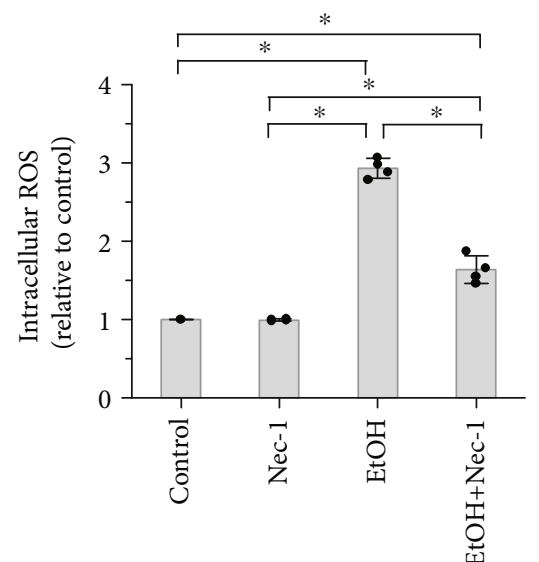

(f)

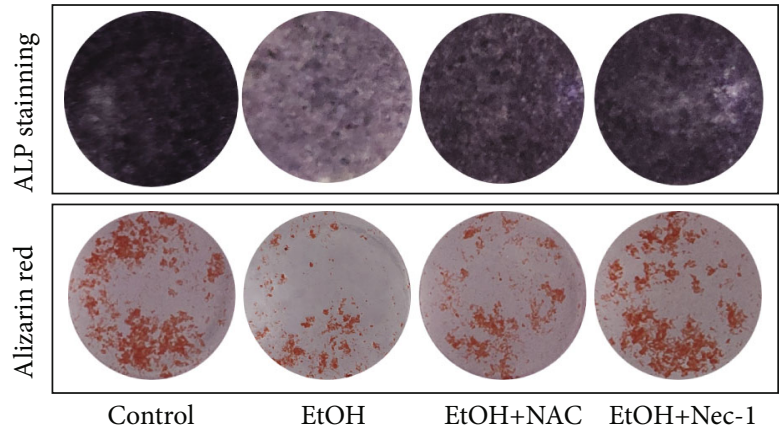

(h)

Figure 6: Continued. 


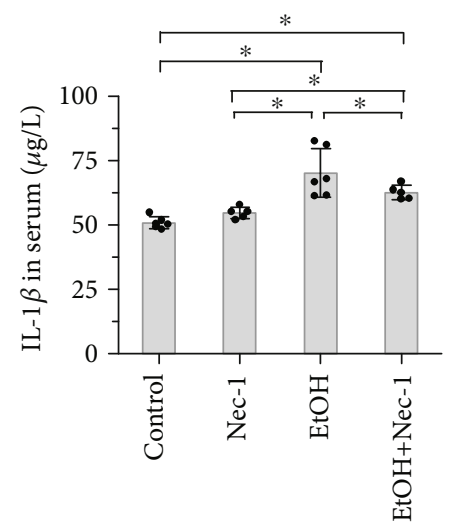

(i)

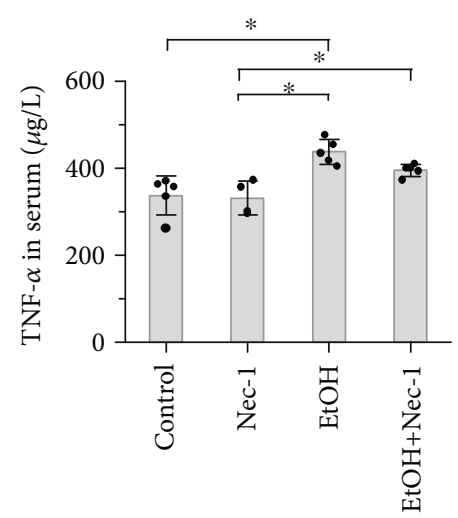

(k)

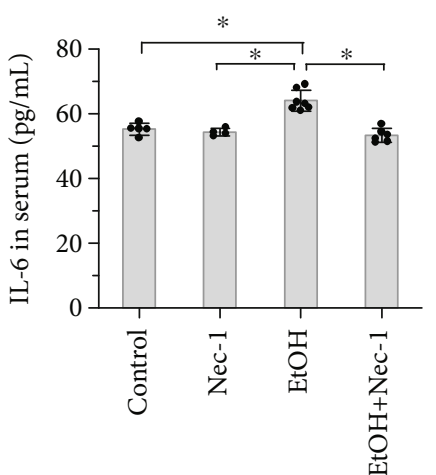

(j)

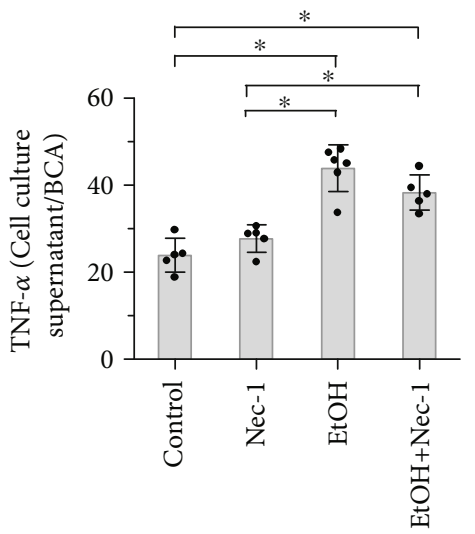

(1)

FIgURE 6: Necrostatin-1 and NAC treatment inhibited the RIPK1/RIPK3/MLKL signaling. (a, b) Western blot analysis showed that EtOH treatment activated the expression levels of the RIPK1 signaling pathway, including p-RIPK1, p-RIPK3, and p-MLKL compared to control mice and MC3T3-E1 cells, and necrostatin-1 treatment lowered the elevated levels both in vivo (a) and in vitro (b). (c, d) Immunohistochemistry staining showed that the expression of RIPK1 in bone tissue increased with EtOH treatment and inhibited by necrostatin-1. The red arrow indicates the positive expression in osteoblasts. Bar: $50 \mu \mathrm{m}$. Confocal laser scanning microscopy revealed that Nec-1 treatment alleviated the increase of RIPK1- and RIPK3-positive osteoblasts in vitro (e). Bar: $20 \mu \mathrm{m}$. (f-h) High dose of EtOH consumption increased the intracellular ROS levels compared with the control group, and Nec-1 directly inhibited the elevated ROS by flow cytometry. The addition of NAC effectively reduced EtOH treatment necroptosis of the osteoblastic cells as NAC attenuated the upregulation of RIPK1 and RIPK3 (g). NAC treatment significantly increased bone formation, as indicated by the increase in ALP staining and the formation of mineralized nodules (alizarin red staining) in osteoblasts $(\mathrm{h}) .(\mathrm{i}, \mathrm{j})$ The levels of serum proinflammatory cytokines (IL-1 $\beta$ and IL-6) were significantly downregulated by Nec-1 treatment compared to the EtOH-treated mice. The level of TNF$\alpha$ elevated in mouse serum (k) and osteoblast culture medium (l) after EtOH treatment by ELISA assay, but had no significant difference after Nec-1 treatment. Error bars represent the SD from the mean values. ${ }^{*} p<0.05$ compared with the control group. ${ }^{\#} p<0.05$ compared with EtOH treatment. TB: trabecular bone; CB: cortical bone; ROS: reactive oxygen species; NAC: N-acetylcysteine; TNF- $\alpha$ : tumor necrosis factor- $\alpha$.

antioxidant NAC to inhibit the production of ROS. In this study, the addition of NAC effectively reduced $\mathrm{EtOH}$ treatment necroptosis of the osteoblastic cells as NAC attenuated the upregulation of RIPK1 and RIPK3 (Figure 6(g)). Meanwhile, NAC treatment significantly increased bone formation, as indicated by the increase in ALP staining and the formation of mineralized nodules (alizarin red staining) in osteoblasts (Figure 6(h)). Together, these results highlight that osteoblast necroptosis, at least in part, promotes the generation of ROS, and on the other hand, ROS play a critical role in the regulation of excessive EtOH treatment-induced osteoblast necroptosis via a positive feedback loop.

\section{Discussion}

Chronic and excessive alcohol consumption causes a reduction in bone mass and destruction of the bone microstructure due to an impairment and/or imbalance in bone remodeling. However, the underlying mechanisms have not yet been elucidated. In this study, we successfully established an alcohol-induced osteopenia mouse model and found that osteopenia is accompanied by decreased bone formation and increased osteoblast necrosis, which was reported in studies on GIOP and ovariectomized rats [40, 41, 47]. Long-term detrimental effects of alcoholism have toxic effects on BMMSCs and affect their differentiation into 
osteoblasts by downregulating the expression of Runx2, which in turn leads to low bone mass $[48,49]$. In addition, previous studies have confirmed that apoptosis was involved in alcohol-induced osteoporosis $[18,50,51]$. Herein, we found that loose trabeculae were more obvious, and the lacunae volume was larger in the EtOH-treated mice than in the control mice. Moreover, some of the MC3T3-E1 treated with alcohol suffered a necrotic change as observed from certain morphological changes and specific markers. These conclusions indicate the involvement of necrosis-associated mechanisms in the osteoblasts treated with EtOH.

Necroptosis is a caspase-independent form of programmed cell death that is triggered by ligation of death receptors or initiators that bind deubiquitylated RIPK1 to autoactivated RIPK3 and initiate the phosphorylation of a pseudokinase substrate MLKL, which then combines with p-RIPK3 to form a necrosome and translocates to the membrane to promote cell lysis $[46,52,53]$. RIPK1 and RIPK3 are key necroptotic effectors that mediate the necroptosis pathway, phosphorylated MLKL is involved in the execution of necroptotic cell death, and caspase-8 is a key factor regulating apoptosis or necroptosis [54]. In this study, we found that the RIPK1/RIPK3/MLKL pathway was involved in alcohol-induced bone disorders and osteoblast necroptosis both in vivo and in vitro. However, we found that the expression of caspase- 8 was not significantly downregulated or inhibited, and the protein levels of cleaved caspase-3, a specific marker of apoptosis, increased in the EtOH-treated group without statistically significant difference. Thus, both necroptosis and apoptosis contributed to alcohol-mediated osteopenia, and necroptosis performed the main inducement. The combination of necroptosis and apoptosis also occurred simultaneously in the process of dexamethasoneinduced MC3T3-E1 cell death [55] and affected the outcomes in liver diseases [56] and other inflammation or tissue damage [57-59].

Studies have revealed important functions and regulatory mechanisms of RIPK1 in inflammation and have indicated the pathological roles of RIPK1 in many human diseases [60]. For noninfectious diseases, inhibition of the RIPK1-dependent necroptosis pathway can ameliorate ischemia-reperfusion injury in the brain, kidney, and heart [61] and alleviate retinal degeneration, brain impact trauma, ethanol-induced liver injury, multiple sclerosis, and tumor progression [37, 62]. Others, TNF-induced systemic inflammatory response syndrome, and even metastasis in cancer cells were also closely related to RIPK1 kinase activation [63]. For infectious diseases, not only virus infections including influenza and HIV but also bacterial infections cause necroptosis-mediated inflammatory responses [64]. It indicated RIPK1 kinase may be a therapeutic target for multiple human diseases. However, it remains unclear whether blocking RIPK1 kinase by necrostatin-1 could repair the damage caused by alcohol-induced osteopenia. Therefore, we administered necrostatin-1 to EtOH-treated mice, BMMSCs, and MC3T3-E1, which can specifically target RIPK1 kinase and block the formation of the necrosome. It is reported that necrostatin- 1 suppresses cell death triggered by caspase inhibition and has no effect on apoptosis $[29,65]$. Our research suggested that necrostatin-1 significantly reduced necrosis of osteoblast induced by alcohol as well as the decreased expression of necroptosis markers RIPK1, RIPK3, and MLKL and blocked/reduced inflammation release as detected by the proinflammatory cytokines (IL- $1 \beta$ and IL6) in mouse serum (Figures 6(i) and 6(j)). The thinned trabecular recovered partially, the levels and capability of osteogenic differentiation from BMMSCs improved, and the expression of the bone formation markers significantly increased after necrostatin-1 treatment. These results suggest that necrostatin-1 directly inhibits the expression of RIPK1, affects the activation of osteoblast necroptosis, and promotes the survival and proliferation of BMMSCs in the presence of death receptor ligands [28], eventually leading to a reduction in osteoblast necrosis and increased bone formation. Consequently, we speculate that necroptosis may be partly involved in the pathological processes underlying alcohol-induced osteopenia and that RIPK1 kinase may be an effective therapeutic target for alcoholinduced osteopenia [40].

Studies revealed that necroptosis was involved in the regulated cell death induced by TNF- $\alpha$ in nonalcoholic fatty liver disease [66], osteoblasts [67], glycerol-induced acute kidney injury [68], etc. However, the specific regulatory mechanism of TNF- $\alpha$ on bone cells and the molecular mechanism in the pathogenesis of osteoporosis remain unclear. It has been reported that TNF- $\alpha$ plays a role in promoting alcohol-induced osteoporosis $[69,70]$. Given that necroptosis is a special type of necrosis mainly mediated by the stimulating death receptor of TNF- $\alpha[27,71]$, we examined the level of TNF- $\alpha$ in mouse serum and osteoblastic culture medium after $\mathrm{EtOH}$ intervention and Nec-1 treatment by ELISA. Both serum and medium contained elevated levels of TNF- $\alpha$ in the EtOH-treated mice and cells but had no significant difference after Nec-1 treatment (Figures $6(\mathrm{k})$ and 6(l)). Therefore, we conjectured that alcohol could induce necroptosis by activating the expression of TNF- $\alpha$ and downstream molecules. Unfortunately, we could not prove the role of TNF- $\alpha$ in the process of EtOH-induced necroptosis. Moreover, detecting the RIPK1/RIPK3/MLKL axis by treating osteoblasts with TNF- $\alpha$ plus the specific inhibitor of necroptosis with or without Nec-1 is necessary to validate that chronic alcohol conditions promote RIPK1 activation with TNF signaling.

ROS have been considered as a driving force for necroptosis, the mitochondrial ROS derived in response to the TNF/TNFR1 pathway [72], and nonmitochondrial ROS generated via TNF-induced necroptosis [73]. Studies showed that treatment with TNF- $\alpha$ could stimulate ROS production, which enhanced necrosome formation [74]. ROS promote RIPK1 autophosphorylation on serine residue 161 (S161), and three crucial cysteines (cysteines 257, 268, and 586) in RIPK1 are required for sensing ROS to regulate RIPK1. The major function of RIPK1 kinase activity in TNFinduced necroptosis is to autophosphorylate S161 which then forms a functional necrosome [75]. TNF-induced ROS function in a positive feedback to enhance necrosome formation and induce necroptosis, when the necrosome complex was impaired or knockdown the RIPK1 or RIPK3, 


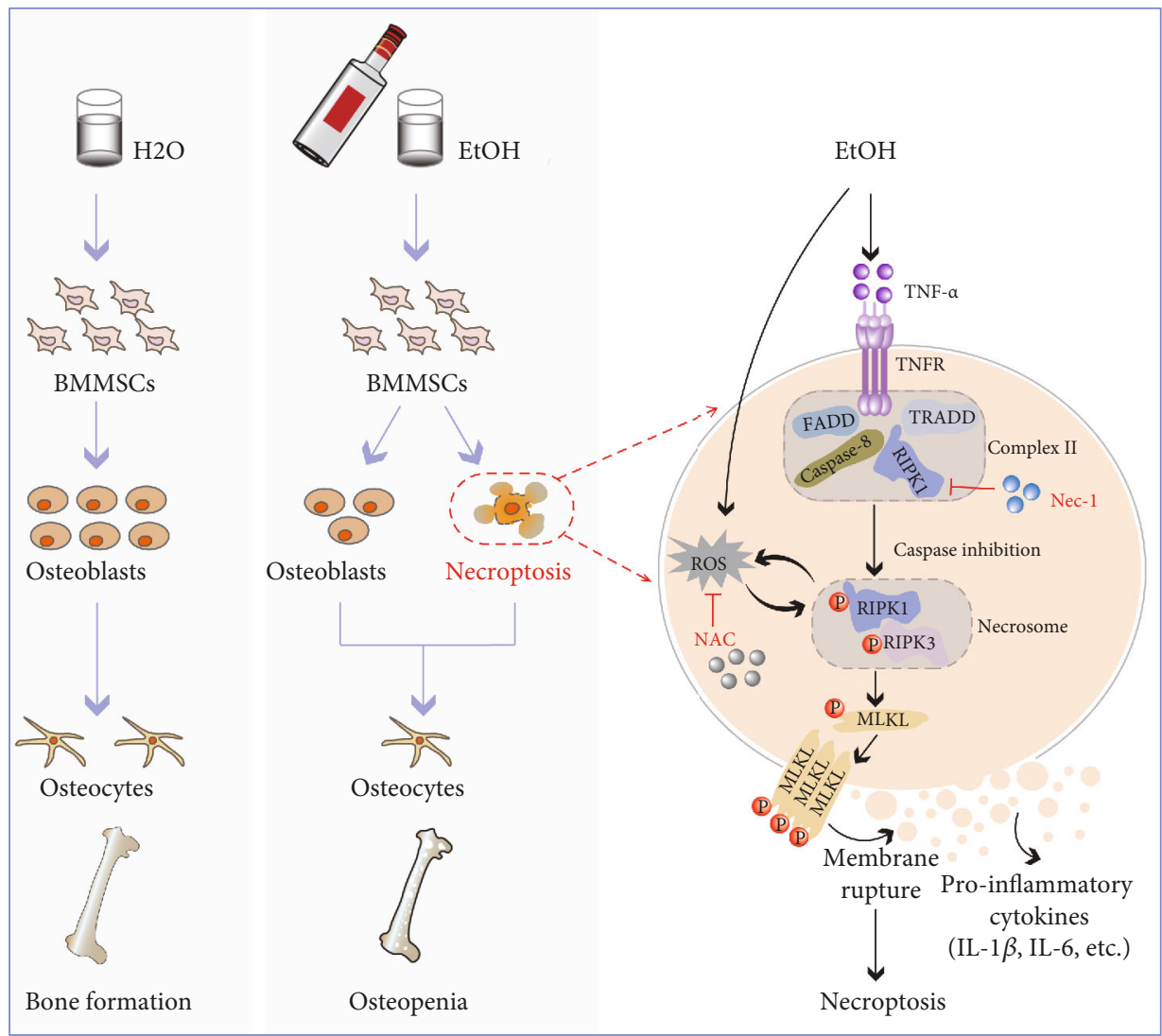

FIGURE 7: EtOH treatment induced osteopenia by increasing osteoblast necroptosis and decreasing BMMSC osteogenic differentiation. Chronic EtOH consumption results in osteopenia and osteoblast necroptosis in mouse model and leads to a necrotic morphology lesion and osteogenic differentiation reduction in bone marrow mesenchymal stem cells (BMMSCs). The ligation is stimulated by EtOH treatment that binds deubiquitylated RIPK1 to autoactivated RIPK3 and initiates the phosphorylation of a pseudokinase substrate MLKL, which then combines with p-RIPK3 to form a necrosome and translocates to the membrane to promote cell lysis and inflammation release (IL-1 $\beta$ and IL-6). Excessive EtOH treatment-induced osteoblast necroptosis might partly depend on ROS generation; concomitantly, ROS contributed to necroptosis of osteoblasts through a positive feedback loop involving RIPK1/RIPK3. Nec-1 and NAC inhibit the expression of RIPK1, affect the activation of necroptosis, and promote the survival and proliferation of osteoblast in the presence of death receptor ligands, eventually leading to a reduction in osteoblast necrosis and increased bone formation. TNFR: tumor necrosis factor receptor; FADD: Fas-associated protein with death domain; TRADD: TNFR1-associated death domain.

led to a decrease in ROS production [74]. In our study, alcohol treatment increased the production of intracellular ROS levels and necrosome complex. Conversely, treatment with Nec-1 markedly suppressed necroptosis and ROS production in EtOH intervention osteoblasts. Altogether, osteoblast necroptosis partly promotes the generation of ROS; on the other hand, ROS contributed to necroptosis of osteoblasts through a positive feedback loop involving RIPK1/RIPK3.

We first demonstrated that the necroptotic pathway underlies alcohol-induced osteopenia and provides evidence to explore a new therapeutic target in mice (Figure 7). Necrostatin-1 treatment successfully treated osteopenia and had long-term therapeutic inhibition in mice due to chronic heavy alcohol consumption. Generally speaking, necrostatin-1 is relatively safe from the current animal experimental observation, but the therapeutic safety and effect of necrostatin-1 on humans require investigation in future studies, including preclinical tests and clinical trials. Other programmed cell deaths, such as apoptosis, autophagy, and pyroptosis, play crucial roles in metabolic processes. Exploring the molecular mechanism in the pathogenesis of AOP, developing selective, potent, and safe biomarkers or inhibitors to measure and treat alcohol-induced osteoblast death remains a key challenge in clinical development.

\section{Conclusions}

In summary, this study provides evidence that excessive EtOH treatment leads to activation of the RIPK1/RIPK3/MLKL signaling, promotes the generation of ROS, resulting in an increase in osteoblast necroptosis and a decrease in BMMSC osteogenic differentiation both in vivo and in vitro, and causes bone mass reduction. Necrostatin1-mediated treatment effectively inhibits the RIPK1/RIPK3/MLKL-dependent signaling, and NAC partly prevents the production of ROS and decreases the activation of necroptosis through a positive feedback loop involving RIPK1/RIPK3 and finally ameliorates alcohol-induced osteopenia by reducing osteoblast necroptosis. Collectively, 
we demonstrated that chronic high-dose alcohol consumption induces osteopenia via necroptosis and thereby discovered that RIPK1 kinase may be a therapeutic target for alcohol-induced osteopenia. However, the detailed mechanism and other molecules involved need to be further investigated.

\section{Data Availability}

The original data used to support the findings of this study are available from the corresponding authors upon request.

\section{Conflicts of Interest}

The authors declare that they have no conflict of interest.

\section{Authors' Contributions}

M. G. and Y. X. participated in the study conception and design. M. G., Y. H., Z. J., and F. T. performed the cell culture, qPCR, and signaling studies. M. G., Q. W., L. C., and $\mathrm{X}$. T. designed and performed the animal experiments. M. G. and S. W. scanned the specimens with the microcomputed tomography (micro-CT) scanner. Q. W., Y. L., Y. Z., and J. G. prepared the figures and performed data analysis. M. G., F. T., and Y. X. prepared and revised the manuscript. Man Guo, Yong-Li Huang, and Qi Wu contributed equally to this work.

\section{Acknowledgments}

We thank Dr. Tao Li and Mr. Jun-Sheng Chen from the West China Hospital of Sichuan University for generating the micro-CT images. This work was supported by grants from the National Natural Science Foundation of China (Grant Number 81970676) and the key projects of Sichuan Science and Technology Department (Grant Numbers 2019YFS0537 and 2020YFS0456).

\section{Supplementary Materials}

Table S1: DNA primers used in quantitative real-time PCR. Figure S1: bone resorption in alcohol-induced osteopenia. Figure S2: identified the isolated mouse BMMSCs and osteoblast. Figure S3: CCK-8 assay was performed to determine the cell proliferation ability. Figure S4: necrostatin-1 treatment inhibited the RIPK1/RIPK3/MLKL signaling. (Supplementary Materials)

\section{References}

[1] G. Freund, "The interaction of chronic alcohol consumption and aging on brain structure and function," Alcoholism, Clinical and Experimental Research, vol. 6, no. 1, pp. 13-21, 1982.

[2] G. Corrao, L. Rubbiati, V. Bagnardi, A. Zambon, and K. Poikolainen, "Alcohol and coronary heart disease: a metaanalysis,” Addiction, vol. 95, no. 10, pp. 1505-1523, 2000.

[3] M. Roerecke, A. Vafaei, O. S. M. Hasan et al., "Alcohol consumption and risk of liver cirrhosis: a systematic review and meta-analysis," The American Journal of Gastroenterology, vol. 114, no. 10, pp. 1574-1586, 2019.

[4] D. A. Chakkalakal, "Alcohol-induced bone loss and deficient bone repair," Alcoholism, Clinical and Experimental Research, vol. 29, no. 12, pp. 2077-2090, 2005.

[5] J. S. Walsh and R. Eastell, "Osteoporosis in men," Nature Reviews. Endocrinology, vol. 9, no. 11, pp. 637-645, 2013.

[6] D. D. Bikle, H. K. Genant, C. Cann, R. R. Recker, B. P. Halloran, and G. J. Strewler, "Bone disease in alcohol abuse," Annals of Internal Medicine, vol. 103, no. 1, pp. 42-48, 1985.

[7] R. T. Turner, "Skeletal response to alcohol," Alcoholism, Clinical and Experimental Research, vol. 24, no. 11, pp. 1693-1701, 2000.

[8] D. B. Maurel, N. Boisseau, C. L. Benhamou, and C. Jaffre, "Alcohol and bone: review of dose effects and mechanisms," Osteoporosis International, vol. 23, no. 1, pp. 1-16, 2012.

[9] A. Diez-Ruiz, P. L. Garcia-Saura, P. Garcia-Ruiz, J. L. Gonzalez-Calvin, F. Gallego-Rojo, and D. Fuchs, "Bone mineral density, bone turnover markers and cytokines in alcohol-induced cirrhosis," Alcohol and Alcoholism, vol. 45, no. 5, pp. 427-430, 2010.

[10] K. E. Friday and G. A. Howard, "Ethanol inhibits human bone cell proliferation and function in vitro," Metabolism, vol. 40, no. 6, pp. 562-565, 1991.

[11] Y. Wang, Y. Li, K. Mao, J. Li, Q. Cui, and G. J. Wang, “Alcoholinduced adipogenesis in bone and marrow: a possible mechanism for osteonecrosis," Clinical Orthopaedics and Related Research, vol. 410, pp. 213-224, 2003.

[12] Y. Liu, X. Kou, C. Chen et al., "Chronic high dose alcohol induces osteopenia via activation of mTOR signaling in bone marrow mesenchymal stem cells," Stem Cells, vol. 34, no. 8, pp. 2157-2168, 2016.

[13] J. R. Chen, O. P. Lazarenko, K. Shankar, M. L. Blackburn, T. M. Badger, and M. J. Ronis, "A role for ethanol-induced oxidative stress in controlling lineage commitment of mesenchymal stromal cells through inhibition of $\mathrm{Wnt} / \beta$-catenin signaling," Journal of Bone and Mineral Research, vol. 25, no. 5, pp. 1117-1127, 2010.

[14] L. F. Bonewald and M. L. Johnson, "Osteocytes, mechanosensing and Wnt signaling," Bone, vol. 42, no. 4, pp. 606-615, 2008.

[15] M. J. Duryee, A. Dusad, C. D. Hunter et al., "N-Acetyl cysteine treatment restores early phase fracture healing in ethanol-fed rats," Alcoholism, Clinical and Experimental Research, vol. 42, no. 7, pp. 1206-1216, 2018.

[16] K. Shankar, M. Hidestrand, X. Liu et al., "Chronic ethanol consumption inhibits postlactational anabolic bone rebuilding in female rats," Journal of Bone and Mineral Research, vol. 23, no. 3, pp. 338-349, 2008.

[17] J. D. Calder, L. Buttery, P. A. Revell, M. Pearse, and J. M. Polak, "Apoptosis-a significant cause of bone cell death in osteonecrosis of the femoral head," Journal of Bone and Joint Surgery. British Volume (London), vol. 86, no. 8, pp. 1209-1213, 2004.

[18] D. B. Maurel, C. Jaffre, G. Y. Rochefort et al., "Low bone accrual is associated with osteocyte apoptosis in alcoholinduced osteopenia," Bone, vol. 49, no. 3, pp. 543-552, 2011.

[19] A. M. Marchini, L. L. Gonçalves, M. C. M. Salgado et al., "Alcoholic and isocaloric diet, but not ovariectomy, influence the apoptosis of bone cells within the alveolar bone crest of rats," Archives of Oral Biology, vol. 59, no. 4, pp. 424-433, 2014. 
[20] C. Gunther, E. Martini, N. Wittkopf et al., "Caspase-8 regulates TNF- $\alpha$-induced epithelial necroptosis and terminal ileitis," Nature, vol. 477, no. 7364, pp. 335-339, 2011.

[21] B. Tummers and D. R. Green, "Caspase-8: regulating life and death," Immunological Reviews, vol. 277, no. 1, pp. 76-89, 2017.

[22] M. Fritsch, S. D. Gunther, R. Schwarzer et al., "Caspase- 8 is the molecular switch for apoptosis, necroptosis and pyroptosis," Nature, vol. 575, no. 7784, pp. 683-687, 2019.

[23] S. Jouan-Lanhouet, M. I. Arshad, C. Piquet-Pellorce et al., "TRAIL induces necroptosis involving RIPK1/RIPK3-dependent PARP-1 activation," Cell Death and Differentiation, vol. 19, no. 12, pp. 2003-2014, 2012.

[24] S. Saveljeva, S. L. Mc Laughlin, P. Vandenabeele, A. Samali, and M. J. Bertrand, "Endoplasmic reticulum stress induces ligand-independent TNFR1-mediated necroptosis in L929 cells," Cell Death \& Disease, vol. 6, no. 1, article e1587, 2015.

[25] N. Holler, R. Zaru, O. Micheau et al., "Fas triggers an alternative, caspase-8-independent cell death pathway using the kinase RIP as effector molecule," Nature Immunology, vol. 1, no. 6, pp. 489-495, 2000.

[26] L. Galluzzi, O. Kepp, F. K. Chan, and G. Kroemer, "Necroptosis: mechanisms and relevance to disease," Annual Review of Pathology, vol. 12, no. 1, pp. 103-130, 2017.

[27] D. E. Christofferson and J. Yuan, "Necroptosis as an alternative form of programmed cell death," Current Opinion in Cell Biology, vol. 22, no. 2, pp. 263-268, 2010.

[28] A. Degterev, D. Ofengeim, and J. Yuan, “Targeting RIPK1 for the treatment of human diseases," Proceedings of the National Academy of Sciences of the United States of America, vol. 116, no. 20, pp. 9714-9722, 2019.

[29] A. Degterev, J. Hitomi, M. Germscheid et al., "Identification of RIP1 kinase as a specific cellular target of necrostatins," Nature Chemical Biology, vol. 4, no. 5, pp. 313-321, 2008.

[30] L. Mifflin, D. Ofengeim, and J. Yuan, "Receptor-interacting protein kinase 1 (RIPK1) as a therapeutic target," Nature Reviews. Drug Discovery, vol. 19, no. 8, pp. 553-571, 2020.

[31] X. X. Deng, S. S. Li, and F. Y. Sun, "Necrostatin-1 prevents necroptosis in brains after ischemic stroke via inhibition of RIPK1-mediated RIPK3/MLKL signaling," Aging and Disease, vol. 10, no. 4, pp. 807-817, 2019.

[32] A. Iannielli, S. Bido, L. Folladori et al., "Pharmacological inhibition of necroptosis protects from dopaminergic neuronal cell death in Parkinson's disease models," Cell Reports, vol. 22, no. 8, pp. 2066-2079, 2018.

[33] H. H. Chan, R. Y. Koh, C. L. Lim, and C. O. Leong, "Receptorinteracting protein kinase 1 (RIPK1) as a potential therapeutic target: an overview of its possible role in the pathogenesis of Alzheimer's disease," Current Alzheimer Research, vol. 16, no. 10, pp. 907-918, 2019.

[34] D. Karunakaran, M. Geoffrion, L. Wei et al., "Targeting macrophage necroptosis for therapeutic and diagnostic interventions in atherosclerosis," Science Advances, vol. 2, no. 7, article e1600224, 2016.

[35] M. I. Oerlemans, J. Liu, F. Arslan et al., "Inhibition of RIP1dependent necrosis prevents adverse cardiac remodeling after myocardial ischemia-reperfusion in vivo," Basic Research in Cardiology, vol. 107, no. 4, p. 270, 2012.

[36] C. C. Smith, S. M. Davidson, S. Y. Lim, J. C. Simpkin, J. S. Hothersall, and D. M. Yellon, "Necrostatin: a potentially novel cardioprotective agent?," Cardiovascular Drugs and Therapy, vol. 21, no. 4, pp. 227-233, 2007.

[37] L. Duprez, N. Takahashi, F. Van Hauwermeiren et al., "RIP kinase-dependent necrosis drives lethal systemic inflammatory response syndrome," Immunity, vol. 35, no. 6, pp. 908 918, 2011.

[38] A. Linkermann, J. H. Brasen, N. Himmerkus et al., "Rip1 (receptor-interacting protein kinase 1) mediates necroptosis and contributes to renal ischemia/reperfusion injury," Kidney International, vol. 81, no. 8, pp. 751-761, 2012.

[39] S. J. Gilbert, S. K. Singhrao, I. M. Khan et al., "Enhanced tissue integration during cartilage repair in vitro can be achieved by inhibiting chondrocyte death at the wound edge," Tissue Engineering. Part $A$, vol. 15, no. 7, pp. 1739-1749, 2009.

[40] M. Feng, R. Zhang, F. Gong et al., "Protective effects of necrostatin-1 on glucocorticoid-induced osteoporosis in rats," The Journal of Steroid Biochemistry and Molecular Biology, vol. 144, no. Part B, pp. 455-462, 2014.

[41] H. Cui, Y. Zhu, Q. Yang et al., "Necrostatin-1 treatment inhibits osteocyte necroptosis and trabecular deterioration in ovariectomized rats," Scientific Reports, vol. 6, no. 1, p. 33803, 2016.

[42] J. Li, Z. Yang, Y. Li et al., "Cell apoptosis, autophagy and necroptosis in osteosarcoma treatment," Oncotarget, vol. 7, no. 28, pp. 44763-44778, 2016.

[43] A. M. Wood, S. Kaptoge, A. S. Butterworth et al., "Risk thresholds for alcohol consumption: combined analysis of individual- participant data for 599912 current drinkers in 83 prospective studies," Lancet, vol. 391, no. 10129, pp. 15131523, 2018.

[44] J. J. Callaci, R. Himes, K. Lauing, F. H. Wezeman, and $\mathrm{K}$. Brownson, "Binge alcohol-induced bone damage is accompanied by differential expression of bone remodeling-related genes in rat vertebral bone," Calcified Tissue International, vol. 84, no. 6, pp. 474-484, 2009.

[45] D. W. Dempster, J. E. Compston, M. K. Drezner et al., "Standardized nomenclature, symbols, and units for bone histomorphometry: a 2012 update of the report of the ASBMR Histomorphometry Nomenclature Committee," Journal of Bone and Mineral Research, vol. 28, no. 1, pp. 2-17, 2013.

[46] M. Pasparakis and P. Vandenabeele, "Necroptosis and its role in inflammation," Nature, vol. 517, no. 7534, pp. 311-320, 2015.

[47] L. Dalle Carbonare, M. E. Arlot, P. M. Chavassieux, J. P. Roux, N. R. Portero, and P. J. Meunier, "Comparison of trabecular bone microarchitecture and remodeling in glucocorticoidinduced and postmenopausal osteoporosis," Journal of Bone and Mineral Research, vol. 16, no. 1, pp. 97-103, 2001.

[48] P. Bianco, M. Riminucci, S. Gronthos, and P. G. Robey, "Bone marrow stromal stem cells: nature, biology, and potential applications," Stem Cells, vol. 19, no. 3, pp. 180-192, 2001.

[49] M. F. Pittenger, A. M. Mackay, S. C. Beck et al., "Multilineage potential of adult human mesenchymal stem cells," Science, vol. 284, no. 5411, pp. 143-147, 1999.

[50] D. B. Maurel, D. Benaitreau, C. Jaffre et al., "Effect of the alcohol consumption on osteocyte cell processes: a molecular imaging study," Journal of Cellular and Molecular Medicine, vol. 18, no. 8, pp. 1680-1693, 2014.

[51] D. B. Maurel, S. Pallu, C. Jaffre et al., "Osteocyte apoptosis and lipid infiltration as mechanisms of alcohol-induced bone loss," Alcohol and Alcoholism, vol. 47, no. 4, pp. 413-422, 2012. 
[52] J. Li, T. McQuade, A. B. Siemer et al., "The RIP1/RIP3 necrosome forms a functional amyloid signaling complex required for programmed necrosis," Cell, vol. 150, no. 2, pp. 339-350, 2012.

[53] Y. Dondelinger, W. Declercq, S. Montessuit et al., "MLKL compromises plasma membrane integrity by binding to phosphatidylinositol phosphates," Cell Reports, vol. 7, no. 4, pp. 971-981, 2014.

[54] K. Newton, K. E. Wickliffe, D. L. Dugger et al., "Cleavage of RIPK1 by caspase- 8 is crucial for limiting apoptosis and necroptosis," Nature, vol. 574, no. 7778, pp. 428-431, 2019.

[55] M. Feng, R. Zhang, P. Yang, K. Wang, and H. Qiang, "Interaction between necroptosis and apoptosis in MC3T3-E1 cell death induced by dexamethasone," Nan Fang Yi Ke Da Xue Xue Bao, vol. 39, no. 9, pp. 1030-1037, 2019.

[56] R. F. Schwabe and T. Luedde, "Apoptosis and necroptosis in the liver: a matter of life and death," Nature Reviews. Gastroenterology \& Hepatology, vol. 15, no. 12, pp. 738-752, 2018.

[57] L. Vasilikos, L. M. Spilgies, J. Knop, and W. W. Wong, "Regulating the balance between necroptosis, apoptosis and inflammation by inhibitors of apoptosis proteins," Immunology and Cell Biology, vol. 95, no. 2, pp. 160-165, 2017.

[58] Y. Xu, H. Gao, Y. Hu et al., "High glucose-induced apoptosis and necroptosis in podocytes is regulated by UCHL1 via RIPK1/RIPK3 pathway," Experimental Cell Research, vol. 382, no. 2, article 111463, 2019.

[59] D. Ruhl, T. T. Du, E. L. Wagner et al., "Necroptosis and apoptosis contribute to cisplatin and aminoglycoside ototoxicity," The Journal of Neuroscience, vol. 39, no. 15, pp. 2951-2964, 2019.

[60] R. Weinlich, A. Oberst, H. M. Beere, and D. R. Green, "Necroptosis in development, inflammation and disease," Nature Reviews. Molecular Cell Biology, vol. 18, no. 2, pp. 127-136, 2017.

[61] A. Linkermann, J. H. Brasen, M. Darding et al., “Two independent pathways of regulated necrosis mediate ischemiareperfusion injury," Proceedings of the National Academy of Sciences of the United States of America, vol. 110, no. 29, pp. 12024-12029, 2013.

[62] D. Ofengeim, Y. Ito, A. Najafov et al., "Activation of necroptosis in multiple sclerosis," Cell Reports, vol. 10, no. 11, pp. 1836$1849,2015$.

[63] B. Strilic, L. Yang, J. Albarran-Juarez et al., "Tumour-cellinduced endothelial cell necroptosis via death receptor 6 promotes metastasis," Nature, vol. 536, no. 7615, pp. 215-218, 2016.

[64] S. Li, L. Zhang, Q. Yao et al., "Pathogen blocks host death receptor signalling by arginine GlcNAcylation of death domains," Nature, vol. 501, no. 7466, pp. 242-246, 2013.

[65] P. Vandenabeele, W. Declercq, F. Van Herreweghe, and T. Vanden Berghe, "The role of the kinases RIP1 and RIP3 in TNF-induced necrosis," Science Signaling, vol. 3, no. 115, 2010.

[66] M. B. Afonso, P. M. Rodrigues, T. Carvalho et al., "Necroptosis is a key pathogenic event in human and experimental murine models of non-alcoholic steatohepatitis," Clinical Science (London, England), vol. 129, no. 8, pp. 721-739, 2015.

[67] G. Shi, P. Jia, H. Chen, L. Bao, F. Feng, and H. Tang, "Necroptosis occurs in osteoblasts during tumor necrosis factor- $\alpha$ stimulation and caspase- 8 inhibition," Brazilian Journal of Medical and Biological Research, vol. 52, no. 1, article e7844, 2018.
[68] R. N. Abd-Ellatif, I. I. Hegab, M. M. Atef, M. T. Sadek, and Y. M. Hafez, "Diacerein protects against glycerol-induced acute kidney injury: modulating oxidative stress, inflammation, apoptosis and necroptosis," Chemico-Biological Interactions, vol. 306, pp. 47-53, 2019.

[69] M. C. Wagner, J. M. Rocha, E. J. Gaio, J. Cavagni, V. C. Carrard, and C. K. Rosing, "Effect of 15\% alcohol dependence on alveolar bone loss and TNF- $\alpha$ secretion in Wistar rats," Brazilian Dental Journal, vol. 27, no. 2, pp. 135-140, 2016.

[70] E. C. Wahl, D. S. Perrien, J. Aronson et al., "Ethanol-induced inhibition of bone formation in a rat model of distraction osteogenesis: a role for the tumor necrosis factor signaling axis," Alcoholism, Clinical and Experimental Research, vol. 29, no. 8, pp. 1466-1472, 2005.

[71] J. Silke, J. A. Rickard, and M. Gerlic, "The diverse role of RIP kinases in necroptosis and inflammation," Nature Immunology, vol. 16, no. 7, pp. 689-697, 2015.

[72] Y. Lin, S. Choksi, H. M. Shen et al., "Tumor necrosis factorinduced nonapoptotic cell death requires receptor-interacting protein-mediated cellular reactive oxygen species accumulation*," The Journal of Biological Chemistry, vol. 279, no. 11, pp. 10822-10828, 2004.

[73] Y. S. Kim, M. J. Morgan, S. Choksi, and Z. G. Liu, “TNFinduced activation of the Nox1 NADPH oxidase and its role in the induction of necrotic cell death," Molecular Cell, vol. 26, no. 5, pp. 675-687, 2007.

[74] B. Schenk and S. Fulda, "Reactive oxygen species regulate Smac mimetic/TNF $\alpha$-induced necroptotic signaling and cell death," Oncogene, vol. 34, no. 47, pp. 5796-5806, 2015.

[75] Y. Zhang, S. S. Su, S. Zhao et al., "RIP1 autophosphorylation is promoted by mitochondrial ROS and is essential for RIP3 recruitment into necrosome," Nature Communications, vol. 8, no. 1, p. 14329, 2017. 